\title{
On the theory of MAG waves and a comparison with sunspot observations from CDS/SoHO
}

\author{
D. Banerjee ${ }^{1}$, E. O'Shea ${ }^{2}$, M. Goossens ${ }^{1}$, J. G. Doyle ${ }^{3}$, and S. Poedts ${ }^{1}$ \\ ${ }^{1}$ Centre for Plasma Astrophysics, Katholieke Universiteit Leuven, Celestijnenlaan 200B, 3001 Leuven, Belgium \\ 2 Instituto de Astrofisica de Canarias, C/ Vía Láctea s/n, 38200 La Laguna, Tenerife, The Canary Islands, Spain \\ 3 Armagh Observatory, College Hill, Armagh BT61 9DG, N. Ireland
}

Received 18 June 2002 / Accepted 23 August 2002

\begin{abstract}
We examine the influence of non-adiabatic effects on the modes of an isothermal stratified magnetic atmosphere. We present new solutions for magneto-acoustic-gravity (or MAG) waves in the presence of a radiative heat exchange based on Newton's law of cooling. An analytic expression for the dispersion relation is derived, which allows the effect of a weak magnetic field on the modes to be studied. The insight so gained proves useful in extending the computations to the moderatehigh field case. In the second part we present observations of two sunspots obtained in the Euv wavelength range with the Coronal Diagnostic Spectrometer (cDs) on SoHO. We examine the time series for the line intensities and relative velocities and calculate their power spectra using wavelet transforms. We find oscillations in the chromosphere and transition region above the sunspots in the temperature range $\log T=4.6-5.4 \mathrm{~K}$. Most of the spectral power above the umbra is contained in the 5-7 mHz frequency range. When the cDs slit crosses the sunspot umbra a clear 3 min oscillation is observed. The observed oscillation frequencies are compared with the computed frequencies and the observations are interpreted in terms of the slow magneto-acoustic waves.
\end{abstract}

Key words. Sun: atmosphere - Sun: magnetic fields - Sun: sunspots - Sun: UV radiation

\section{Introduction}

In the past thirty years, observations of oscillations with periods around 3 min have been widely reported in the atmosphere of sunspots (see reviews by Lites 1992; Bogdan 2000; Fludra 1999, 2001; Brynildsen et al. 1999a,b, 2000, 2002; Maltby et al. 1999, 2001; Tziotziou et al. 2002; O'Shea et al. 2002). It is widely believed that these oscillations are the signatures of waves propagating in the sunspot atmosphere. A study of these oscillations can therefore be used to reveal information about the form of the waves and the structure and nature of the sunspot. The aim of the present study is to contribute towards developing a theory of such wave motions and to compare them with observations performed by the Coronal Diagnostic Spectrometer (CDS) on SoHO. The waves that we consider here are magneto-acoustic-gravity (MAG for short) waves. We study the physical nature of the MAG oscillations and try to understand the cause for the existence of different types of elementary wave modes in a magnetized radiative isothermal atmosphere, subject to different sets of boundary conditions. The present investigation is a continuation of earlier work by Hasan \& Christensen-Dalsgaard (1992) and Banerjee et al. (1995), who examined the effects of a weak vertical magnetic field on the normal adiabatic modes of an isothermal atmosphere

Send offprint requests to: D. Banerjee,

e-mail: dipu@wis.kuleuven.ac.be by combining a semi-analytic approach, based on asymptotic dispersion relations, with numerical solutions. However, oscillations in a realistic solar atmosphere are affected by radiative dissipation and energy losses at the boundaries. Thus the modes are damped and have complex frequencies. In this paper, we examine the influence of non-adiabatic effects on the modes of an isothermal stratified magnetized atmosphere. The inclusion of radiative dissipation based on Newton's law of cooling demonstrates the importance of this effect in the study of magneto-atmospheric waves. It was pointed out by Bünte \& Bogdan (1994) that Newtonian cooling can be incorporated in the solution of the isothermal magneto-atmospheric wave problem by replacing $\gamma$, the ratio of specific heats, by a complex frequency-dependent quantity. This procedure permits one to generalize easily the previous calculations to include radiative dissipation. Bünte \& Bogdan treated a planar, isothermal and stratified atmosphere in the presence of a horizontal magnetic field, whereas in this study we consider a vertical magnetic field. Babaev et al. (1995) derived an exact solution of the MAG waves in the case of oblique propagation with respect to the magnetic field. The solutions are expressed in terms of the generalized Meijer's hypergeometric G functions. Their solutions are very similar to our analytical results. We further perform a normal mode analysis subject to different sets of boundary conditions and compare our analytical results with full numerical solutions. 
We consider wave damping by radiative energy exchange, which is likely to be efficient in the solar photosphere, where the radiative relaxation times are very short compared to the typical wave periods. By comparison, damping of hydromagnetic waves due to viscous dissipation and particle conduction is entirely negligible in those layers of the solar atmosphere where small amplitude disturbances are likely to occur. As we will see later, the condition for the propagation of gravity waves, which depends on the existence of the buoyancy force, is more stringent in the presence of radiative damping than in its absence (Bray \& Loughhead 1974). The radiative damping of oscillatory modes in a optically thin, isothermal, unmagnetized medium was studied by several authors (Stix 1970; Souffrin 1972; Mihalas \& Mihalas 1984). Bogdan \& Knölker (1989) obtained the dispersion relation for linear compressive plane waves in a homogeneous, unstratified, uniformly magnetized radiating fluid. Here we consider the propagation of optically thin MAG waves in a stratified, uniformly magnetized medium in which the radiative energy exchange occurs through Newton's law of cooling. The main effect of radiation is to damp the waves. The plan of the paper is as follows: in Sect. 2, the basic wave equations are presented, including Newton's law of cooling. In Sects. 3 and 4 we present the dispersion relation for a weak field followed by numerical results in the form of a $K-\Omega$ diagram. In Sect. 5 we treat the strong field case which will be more relevant for comparison with our observations. This is followed by an observational report of sunspot oscillation studies by CDS in Sects. 6 and 7. A discussion of the observational results and a comparison with the theoretical results are taken up in Sect. 8 and finally the conclusions are drawn in Sect. 9.

\section{The wave equation with Newtonian cooling}

We confine our attention to an isothermal atmosphere with a vertical magnetic field which is unbounded in the horizontal direction. Using a fluid description and assuming an ideal plasma, we write the energy equation as,

$\partial_{\mathrm{t}} \frac{\delta T}{T}+\delta v_{z} \frac{1}{T} \frac{\mathrm{d} T}{\mathrm{~d} z}+(\gamma-1) \nabla \cdot \delta v=-\frac{1}{\tau_{\mathrm{R}}} \frac{\delta T}{T}$,

where $\delta v=\partial_{\mathrm{t}} \xi$ is the velocity perturbation, $T$ is the temperature of the gas and $\delta T$ the temperature perturbation. We assume the Lagrangian displacement $\xi$ varies as $\sim \mathrm{e}^{\mathrm{i}(\omega t-k x)}$, where $\omega$ is the angular frequency and $k$ is the horizontal wave number. We allow for radiative losses, approximating them by Newton's law of cooling (e.g. Spiegel 1957; Mihalas \& Mihalas 1984), which assumes that the temperature fluctuations are damped radiatively on a time scale $\tau_{\mathrm{R}}$, given by

$\tau_{\mathrm{R}}=\frac{\rho c_{\mathrm{v}}}{16 \chi \sigma T^{3}}$,

where $\chi$ is the mean absorption coefficient per unit length, $c_{\mathrm{v}}$ the specific heat per unit volume, and $\sigma$ the Stefan-Boltzmann constant. For simplicity, we assume that $\tau_{\mathrm{R}}$ is constant over the atmosphere. If the vertical dimension of the perturbation is small compared to the local scale height, the relation between
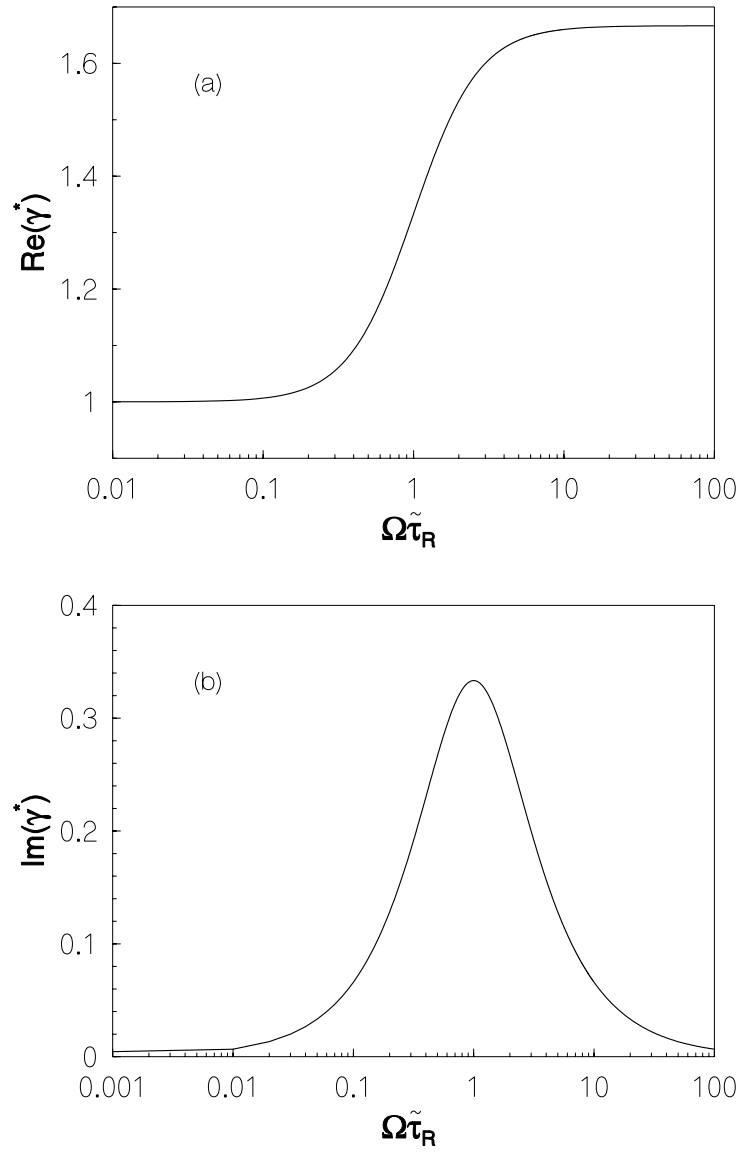

Fig. 1. The complex parameter $\gamma^{*}$ as a function of $\Omega \tilde{\tau}_{R}$. Panels a) represents the variation of the real part and $\mathbf{b}$ ) represents the variation of the imaginary part.

the Lagrangian perturbations in pressure $p$ and density $\rho$ is then approximately given by $\delta p / p \simeq \gamma^{*} \delta \rho / \rho$, where

$\gamma^{*}(\omega)=\frac{1+\mathrm{i} \omega \tau_{\mathrm{R}} \gamma}{1+\mathrm{i} \omega \tau_{\mathrm{R}}}$

With these assumptions, the linearized equations for MAG waves are given by a system of two coupled differential equations (Banerjee et al. 1997),

$\left[v_{\mathrm{A}}^{2} \frac{\mathrm{d}^{2}}{\mathrm{~d} z^{2}}-\left(\tilde{\gamma} c_{\mathrm{S}}^{2}+v_{\mathrm{A}}^{2}\right) k^{2}+\omega^{2}\right] \xi_{x}-\mathrm{i} k\left(\tilde{\gamma} c_{\mathrm{S}}^{2} \frac{\mathrm{d}}{\mathrm{d} z}-g\right) \xi_{z}=0$,

$\left[\tilde{\gamma} c_{\mathrm{S}}^{2} \frac{\mathrm{d}^{2}}{\mathrm{~d} z^{2}}-\tilde{\gamma} \gamma g \frac{\mathrm{d}}{\mathrm{d} z}+\omega^{2}\right] \xi_{z}-\mathrm{i} k\left[\tilde{\gamma} c_{\mathrm{S}}^{2} \frac{\mathrm{d}}{\mathrm{d} z}-(\tilde{\gamma} \gamma-1) g\right] \xi_{x}=0$,

where $\xi_{z}$ and $\xi_{x}$ are the amplitudes of the vertical and horizontal components of the displacement, $g$ is the acceleration due to gravity, and $\tilde{\gamma}=\gamma^{*} / \gamma$. The adiabatic sound speed and the Alfvén speed are given, respectively, by

$c_{\mathrm{S}}=\sqrt{\frac{\gamma p}{\rho}} \quad$ and $\quad v_{\mathrm{A}}=\frac{B}{\sqrt{4 \pi \rho}}$.

We should point out here that the equation governing the propagation of the purely transverse Alfvén waves is decoupled from Eqs. (4) and (5) and will not be considered in the present investigation. We have implicitly assumed that the propagation and 


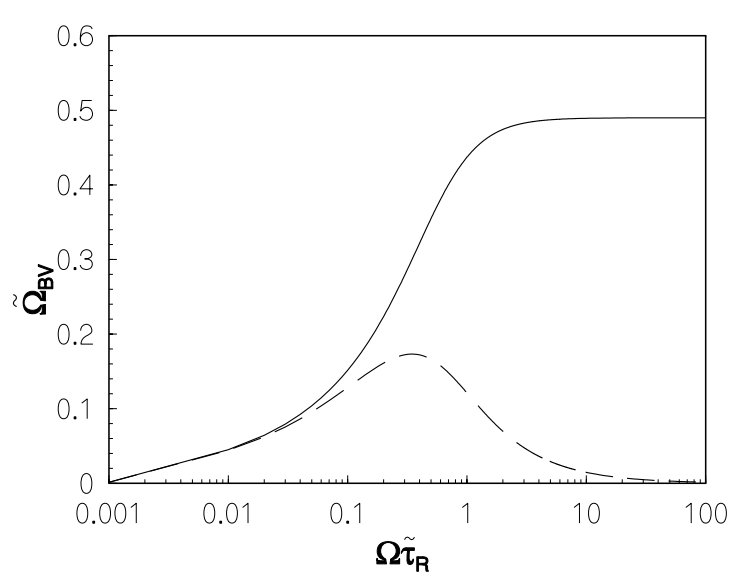

Fig. 2. The variation of the real part (solid line) and the imaginary part (dashed line) of the effective Brunt-Väisälä frequency $\tilde{\Omega}_{\mathrm{BV}}$, (in dimensionless units) as a function of $\Omega \tilde{\tau}_{\mathrm{R}}$.

the motions of the MAG modes are confined to the $x-z$ plane. This involves no loss of generality. Equations (4) and (5) have the same structure as the linearized wave equation for adiabatic perturbations (see Eqs. (1), (2) of Hasan \& ChristensenDalsgaard 1992), apart from the appearance of the parameter $\tilde{\gamma}$, which incorporates radiative cooling. In non-dimensional form Eq. (3) can be written as

$\gamma^{*}=\frac{1+\mathrm{i} \Omega \tilde{\tau}_{\mathrm{R}} \gamma}{1+\mathrm{i} \Omega \tilde{\tau}_{\mathrm{R}}}$

where the dimensionless relaxation time scale is given by $\tilde{\tau}_{\mathrm{R}}=$ $\left(c_{\mathrm{S}} / H\right) \tau_{\mathrm{R}}$, where $H=p / \rho g$ is the scale height of the atmosphere, which is constant for an isothermal medium and the dimensionless frequency, $\Omega$, is given by expression (9). As $\Omega \tilde{\tau}_{R}$ varies from 0 to $\infty, \gamma^{*}$ describes a semi-circle in the complex plane (see Bünte \& Bogdan 1994). Figures 1a, b show the variation of the real and imaginary parts of $\gamma^{*}$ respectively as a function of $\Omega \tilde{\tau}_{\mathrm{R}}$. We find that for $\Omega \tilde{\tau}_{\mathrm{R}}<0.1, \gamma^{*}$ approaches the isothermal limit, and $\gamma^{*}=1$. For $\Omega \tilde{\tau}_{\mathrm{R}}>10, \operatorname{Re}\left(\gamma^{*}\right)=5 / 3=\gamma$. Thus in the limit $\tau_{\mathrm{R}}, \rightarrow \infty$ i.e. in the limit of adiabatic perturbations, $\gamma^{*}=\gamma$ and $\tilde{\gamma}=1$. Letting $\tilde{\gamma}=1$ in Eqs. (4) and (5) we recover the linearized equations given by Hasan \& ChristensenDalsgaard (1992). The imaginary contribution to $\gamma^{*}$ is maximal for $\Omega \tilde{\tau}_{\mathrm{R}} \sim 1$. Thus to study the maximal effect of radiative heat exchange we choose our parametric values such that $\Omega \tilde{\tau}_{R}$ becomes close to 1 . In order to obtain a dimensionless wave equation we introduce three dimensionless parameters,

$K=k H$,

$\Omega=\frac{\omega H}{c_{\mathrm{S}}}$,

and the dimensionless vertical coordinate

$\theta=\frac{\omega H}{v_{\mathrm{A}}}=\frac{c_{\mathrm{S}}}{v_{\mathrm{A}, 0}} \Omega \mathrm{e}^{-z /(2 H)}$, where $v_{A, 0}$ is the Alfvén speed at $z=0$. In terms of the variables defined by Eqs. (8)-(10), Eqs. (4) and (5) can be combined into a fourth-order differential equation for $\xi_{x}$,

$$
\begin{array}{r}
\left\{\theta^{4} \frac{\mathrm{d}^{4}}{\mathrm{~d} \theta^{4}}+4 \theta^{3} \frac{\mathrm{d}^{3}}{\mathrm{~d} \theta^{3}}+\right. \\
+\left[1+4\left(\frac{\Omega^{2}}{\tilde{\gamma}}-K^{2}\right)+4 \theta^{2}\right] \theta^{2} \frac{\mathrm{d}^{2}}{\mathrm{~d} \theta^{2}} \\
\left.+16\left[\left(\frac{\Omega^{2}}{\tilde{\gamma}}+K^{2}\left(\frac{\tilde{\Omega}_{\mathrm{BV}}^{2}}{\Omega^{2}}-1\right)\right) \theta^{2}-\frac{\Omega^{2}}{\tilde{\gamma}}+K^{2}\right)-12 \theta^{2}\right] \theta \frac{\mathrm{d}}{\mathrm{d} \theta} \\
+\xi_{x}=0,
\end{array}
$$

where $\tilde{\Omega}_{\mathrm{BV}}^{2}=\left(\gamma^{*}-1\right) / \gamma \gamma^{*}$ is the square of the effective BruntVäisälä frequency (in dimensionless units). This Brunt-Väisälä frequency is a function of frequency in the non-adiabatic case.

Figure 2 shows the dependence of $\tilde{\Omega}_{\mathrm{BV}}$ on the radiative relaxation time $\tilde{\tau}_{\mathrm{R}}$. The solid line depicts the real part whereas the dashed line represents the imaginary part of $\tilde{\Omega}_{\mathrm{BV}}$. Note that for $\Omega \tilde{\tau}_{\mathrm{R}}>10, \tilde{\Omega}_{\mathrm{BV}}$ reaches a constant value of 0.5 (corresponding to the adiabatic limit). On the other hand, as $\Omega \tilde{\tau}_{R} \rightarrow 0$, $\operatorname{Re}\left(\tilde{\Omega}_{\mathrm{BV}}\right)<0.1$. Thus, in the isothermal limit, $\tilde{\Omega}_{\mathrm{BV}}$, which is the higher cutoff frequency for the $g$-modes, is very low. The consequences of this will be taken up again when we discuss the properties of $g$-modes in detail. Figure 2 also reveals that the imaginary part of $\tilde{\Omega}_{\mathrm{BV}}$ is significant only for $\Omega \tilde{\tau}_{\mathrm{R}} \sim 1$.

The general solution of Eq. (11) can be expressed in terms of Meijer functions (Zhugzhda 1979) as follows:

$\xi_{x}^{(\mathrm{h})}=G_{2,4}^{12}\left(\mu_{h}, \begin{array}{l}a_{1}, \\ \mu_{1}, \cdots, \mu_{i}, \cdots, \mu_{4}\end{array} \mid \theta^{2}\right)$,

where $(i, h=1, \ldots, 4 ; i \neq h)$ and

$\mu_{1,2}=\frac{(1 \pm \mathrm{i} \alpha)}{2}, \quad \mu_{3,4}= \pm K$,

$a_{1,2}=\frac{(1 \pm \phi)}{2}$

$\alpha=\sqrt{4 \frac{\Omega^{2}}{\tilde{\gamma}}-1}$

$\phi=\sqrt{-\alpha^{2}+4 K^{2}\left(1-\tilde{\Omega}_{\mathrm{BV}}^{2} / \Omega^{2}\right)}$

These solutions are very similar in nature to the purely adiabatic case (see Banerjee et al. 1995). Once $\xi_{x}^{(\mathrm{h})}$ is known, it is fairly straightforward to determine the corresponding solutions $\xi_{z}^{(\mathrm{h})}$ from either of Eqs. (4) or (5). The complete solutions satisfying the required boundary conditions can be constructed as linear combinations of $\xi_{x}^{(\mathrm{h})}$ and $\xi_{z}^{(\mathrm{h})}$.

\section{Normal modes in a weak magnetic field}

We now examine the asymptotic properties of waves and normal modes of a stratified atmosphere with a weak magnetic field (corresponding to the limit of small $\epsilon$, where $\epsilon=v_{\mathrm{A}, 0} / c_{\mathrm{S}}$ ). The analytical results are used for the interpretation of the numerical solutions presented in Sect. 4. In order to get a physical picture of the solution, we consider the upward propagation of a wave, excited from below at $z=0$, in an isothermal 

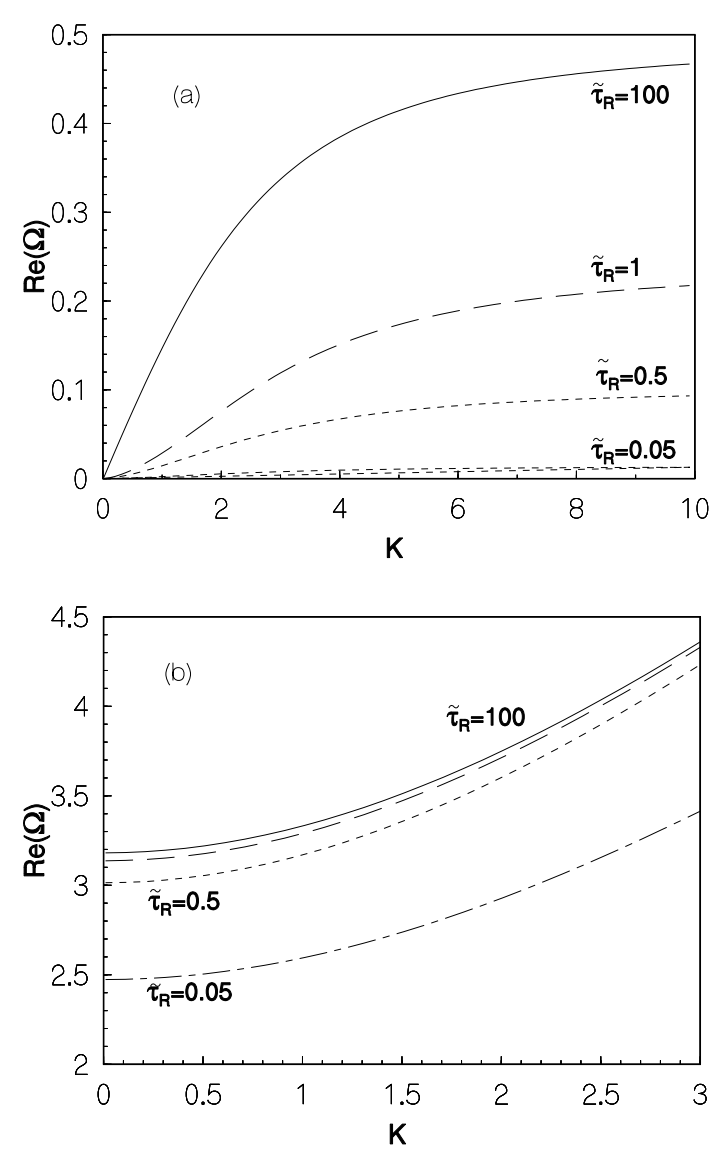

Fig. 3. Diagnostic diagram for non adiabatic modes. Panel a) for $g_{1}-$ mode and $\mathbf{b}$ ) for $p_{1}$-mode. Different line styles correspond to different radiative relaxation time $\tilde{\tau}_{\mathrm{R}}$ as labelled.

atmosphere. It is well known that acoustic modes are easily reflected if the temperature of the medium changes with height (for a good discussion see Leibacher \& Stein 1981). The slow mode can be reflected due to the increasing Alfvén speed with height from layers where $v_{\mathrm{A}} \sim c_{\mathrm{S}}$, through conversion into a fast mode (e.g. Zhugzhda et al. 1984). We implicitly assume that the properties of the atmosphere change abruptly at the top boundary, resulting in downward reflection of the waves. The lower boundary condition is chosen to simulate a forcing layer. This permits standing wave solutions. It should, however, be kept in mind that an isothermal atmosphere by itself does not trap modes, rather we use this assumption to understand the physical properties of the modes in a stratified atmosphere with a vertical field. Let us now derive approximate dispersion relations for various boundary conditions.

\subsection{Rigid boundary condition}

Let us first consider rigid boundary conditions, viz.

$\xi_{x}=\xi_{z}=0$ at $z=0$ and $z=d$,

here $d$ is the height of the upper boundary, and $D=d / H$ is the dimensionless height. The asymptotic properties of the solution in the purely adiabatic limit are presented in Hasan \& Christensen-Dalsgaard (1992). Following the same line of

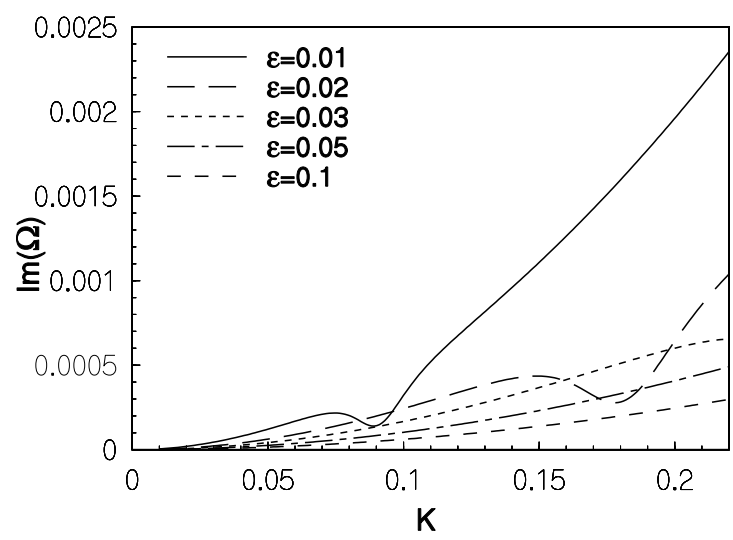

Fig. 4. Variation of the imaginary part of $\Omega$ with $K$ for $\tilde{\tau}_{\mathrm{R}}=0.5$. The different line styles corresponds to different $\epsilon$ values (which is a measure of magnetic field strength) as labelled.

treatment using Eqs. (12)-(16) and applying the boundary conditions given by Eq. (17) one can derive the following dispersion relation in the weak field limit

$$
\begin{aligned}
&\left(\frac{\Omega^{2}}{\tilde{\gamma}}-K^{2}\right) \sin \tilde{\theta} \sin \left(K_{z} D\right) \\
&=2 \sqrt{\tilde{\gamma}} \frac{\epsilon}{\Omega} \mathrm{e}^{D / 4}\left\{K_{z} K^{2}\left[\cosh (D / 4) \cos \tilde{\theta} \cos \left(K_{z} D\right)-1\right]\right. \\
&+\sinh (D / 4) \cos \tilde{\theta} \sin \left(K_{z} D\right)\left[M\left(\frac{\Omega^{2}}{\tilde{\gamma}}-K^{2}\right)\right. \\
&\left.\left.-K^{2}\left(\frac{1}{\gamma \tilde{\gamma}}-\frac{1}{2}\right)\right]\right\}+O\left(\frac{\epsilon^{2}}{\Omega^{2}}\right),
\end{aligned}
$$

where

$\theta_{0}=\theta(0), \quad \theta_{D}=\theta(D), \quad \tilde{\theta}=2\left(\theta_{0}-\theta_{D}\right)$,

and $K_{z}^{2}$ is given by

$K_{z}^{2}=\frac{\Omega^{2}}{\tilde{\gamma}}-K^{2}\left(1-\frac{\tilde{\Omega}_{\mathrm{BV}}^{2}}{\Omega^{2}}\right)-\frac{1}{4}$,

and

$M=K^{2} \frac{\tilde{\Omega}_{\mathrm{BV}}^{2}}{\Omega^{2}}-\frac{1}{16}$.

For $\epsilon<<\Omega$, the dispersion relation to lowest order in $\epsilon / \Omega$ becomes

$\left(\frac{\Omega^{2}}{\tilde{\gamma}}-K^{2}\right) \sin \tilde{\theta} \sin \left(K_{z} D\right)=0$.

Equation (22) admits the following solutions

$\sin \left(K_{z} D\right)=0$,

$\sin \tilde{\theta}=0$,

$\Omega=\sqrt{\tilde{\gamma}} K$.

We first consider the solution given by Eq. (23) which implies that $K_{z} D=n \pi$, where $n$ is an integer and denotes the order of the mode. Using this condition Eq. (20) yields,

$\frac{\Omega_{i}^{4}}{\tilde{\gamma}}-\Omega_{i}^{2}\left(K_{\mathrm{t}}^{2}+\frac{1}{4}\right)+K^{2} \tilde{\Omega}_{\mathrm{BV}}^{2}=0 \quad(i=p, g)$, 
where $K_{t}^{2}=K_{z}^{2}+K^{2}$. Note that Eq. (26) looks very similar to the usual relation for $p-$ and $g$-modes (see Banerjee et al. 1995) apart from the factor $\tilde{\gamma}$ and modified $\tilde{\Omega}_{\mathrm{BV}}$. Because of the presence of these two factors, the properties of these modes change drastically. Figures $3 \mathrm{a}, \mathrm{b}$ give an overview of the behaviour of the $g$ - and $p$-type modes respectively. Their properties are reflected in these two $K-\Omega$ diagrams produced by solving Eq. (26) for different values of the relaxation time $\tilde{\tau}_{\mathrm{R}}$. Figure $3 \mathrm{a}$ clearly shows that the $g_{1}$-mode has been pushed down to the low frequency part of the diagnostic diagram with decreasing value of $\tilde{\tau}_{\mathrm{R}}$. As $\tilde{\tau}_{\mathrm{R}} \rightarrow 0$, the $g_{1}$-mode tends to disappear, because $\tilde{\Omega}_{\mathrm{BV}} \rightarrow 0$. On the other hand, Fig. $3 \mathrm{~b}$ shows that the inclusion of radiative exchange has not greatly changed the behaviour of the $p_{1}$-mode, apart from a decrease of the acoustic cutoff frequency.

The solution corresponding to Eq. (25) can be recognized as a modified Lamb mode (compare with $\Omega=K$, for pure Lamb mode). Thus we expect a frequency shift of the adiabatic Lamb mode. Turning our attention to the solution given by Eq. (24), we find that these modes are the same magnetic modes present in the adiabatic conditions, which arise solely due to the presence of the magnetic field. The magnetic modes, hereafter referred to as $m$-modes, have frequencies

$\Omega_{\mathrm{m}}=\frac{\epsilon l \pi}{2 s}, \quad(l=1,2, \ldots)$,

where $s=\left(1-\mathrm{e}^{-D / 2}\right)$. These modes are approximately transverse (it has be shown by Banerjee et al. (1995) that $\left.\xi_{x}^{(1,2)} / \xi_{z}^{(1,2)} \sim O(\theta)\right)$. Physically, these modes can be interpreted as gravity-modified slow modes in a weak magnetic field. Thus these slow modes are not affected by the inclusion of radiative losses in the weak field limit. This result complements the result of Bogdan \& Knölker (1989), where it was conjectured that the uniform magnetic field reduces the temperature perturbations associated with these waves and therefore suppresses the radiative damping of these disturbances. This aspect will be taken up later when we discuss the numerical solutions.

\subsection{Zero-gradient boundary condition}

If we use zero-gradient boundary conditions at the top and bottom of the layer,

$\frac{\mathrm{d} \xi_{x}}{\mathrm{~d} z}=\frac{\mathrm{d} \xi_{z}}{\mathrm{~d} z}=0 \quad$ at $\quad z=0 \quad$ and $\quad z=d$.

In addition to the modes discussed previously, we find another wave mode, namely the gravity-Lamb mode. The dispersion relation for this mode is given by

$K_{z}^{2}+\frac{1}{4}=0$.

Combining Eqs. (29) with (20) yields

$\Omega^{4}-\tilde{\gamma} \Omega^{2} K^{2}+\tilde{\gamma} K^{2} \Omega_{\mathrm{BV}}^{2}=0$.

This equation has the solution

$\Omega^{2}=\frac{\tilde{\gamma} K^{2}}{2}\left[1 \pm\left(1-4 \frac{\tilde{\Omega}_{\mathrm{BV}}^{2}}{K^{2} \tilde{\gamma}}\right)^{1 / 2}\right]$.
The solution resembles a modified gravity mode on the lower branch and a Lamb mode on the upper branch. In order to see this, consider the limit $K \rightarrow \infty$. The smaller solution in Eq. (31) has the limit $\Omega \simeq \tilde{\Omega}_{\mathrm{BV}}$, which is the dispersion relation for a modified $g$-mode for large $K$; the larger solution has the limit $\Omega \simeq \sqrt{\tilde{\gamma}} K$ for large $K$, which shows that the mode behaves like a modified Lamb wave.

Thus the separate modes have changed their behavior in the diagnostic diagram in the non-adiabatic case. It is important to know how these modified modes interact with one another in the presence of radiative losses. Mode coupling in the non adiabatic case will be different as compared to the adiabatic case studied by Banerjee et al. (1995) (the right hand side of Eq. (18) contributes to the coupling).

\section{Numerical results}

The behavior of the MAG waves is reflected in their properties in the $K-\Omega$ diagram namely the variation of the real and imaginary part of the complex frequency with the horizontal wave number $K$. The solutions were obtained by solving Eq. (11) numerically, using a complex version of the Newton-RaphsonKantorovich scheme (Cash \& Moore 1980) subject to a different sets of boundary conditions. Banerjee et al. (1997) presented the numerical solutions for the weak field case subjected to rigid boundary conditions. In this paper we would like to compare our theoretical results with some observational results so we concentrate here on higher magnetic field strengths.

First we show the effect of the strength of the magnetic field on the damping of these waves. To delineate the influence of the magnetic field, we choose the $m_{2}$-mode, which is predominantly magnetic in nature. Figure 4 shows the variation of the imaginary part of the frequency (which is a measure of the damping) with $K$ for fixed $\tilde{\tau}_{\mathrm{R}}=0.5, D=1$, and $\gamma=5 / 3$ (for rigid boundary conditions). The different line styles correspond to different $\epsilon$ values. It clearly reveals that as we increase the value of $\epsilon$ (increasing magnetic field strength) the imaginary part reduces, indicating less damping of these wave modes. This result is in agreement with the conclusions drawn by Bogdan \& Knölker (1989), that the magnetic field suppresses radiative damping. For horizontal magnetic field Bünte \& Bogdan (1994) also reported a "stiffening" of the atmosphere with increasing $\epsilon$ values.

Let us now consider a moderate magnetic field strength. Figure 5 shows a region in the diagnostic diagram for $\epsilon=0.1$ $(B \sim 240 \mathrm{G})$ and $\tilde{\tau}_{\mathrm{R}}=0.5$, subject to the zero-gradient boundary conditions. The mode coupling in this case is much more complicated because we have three mode interaction regions as indicated. As $K$ increases, the $m_{1}-$ mode begins to acquire the character of a modified Lamb mode. Figure 6a, which shows the variation of imaginary part of the frequency of the $m_{1}-$ mode with $K$ also reveals that, there is an enhancement as it approaches an avoided crossing (near $K=0.8$ ) followed by a suppression due to mode transformation. Up to $K=0.8$ this mode behaves as a magnetic Lamb mode and after the mode transformation it becomes a magnetic type. This process is repeated at higher frequency (around $K=2$ ). Note the large drop due to magnetic field suppression. Figure $6 \mathrm{~b}$ shows the 
Table 1. Eigenfrequencies of different order $p$-modes for a model atmosphere with $D=10, B=2 k G, \tilde{\tau}_{\mathrm{R}}=0.5$ and $K=0.1$.

\begin{tabular}{|c|c|c|c|c|c|c|c|c|c|}
\hline \multirow[b]{2}{*}{ Mode } & \multicolumn{3}{|c|}{$\begin{array}{c}\text { Adiabatic case } \\
\left(\tilde{\tau}_{\mathrm{R}}=100\right)\end{array}$} & \multicolumn{3}{|c|}{$\begin{array}{c}\text { Radiative case } \\
\left(\tilde{\tau}_{\mathrm{R}}=0.5\right)\end{array}$} & \multicolumn{3}{|c|}{$\begin{array}{c}\text { Isothermal case } \\
\left(\tilde{\tau}_{\mathrm{R}}=0.05\right)\end{array}$} \\
\hline & $\operatorname{Re}(\Omega)$ & $\operatorname{Im}(\Omega)$ & $P(\mathrm{~S})$ & $\operatorname{Re}(\Omega)$ & $\operatorname{Im}(\Omega)$ & $P(\mathrm{~S})$ & $\operatorname{Re}(\Omega)$ & $\operatorname{Im}(\Omega)$ & $P(\mathrm{~S})$ \\
\hline$p_{1}$ & 0.5903 & 0.0007 & 164 & 0.5203 & 0.0658 & 186 & 0.458 & 0.0125 & 211 \\
\hline$p_{2}$ & 0.803 & 0.0009 & 120 & 0.7075 & 0.0895 & 136 & 0.624 & 0.017 & 155 \\
\hline$p_{3}$ & 1.07 & 0.0013 & 90 & 0.94 & 0.119 & 103 & 0.828 & 0.0227 & 117 \\
\hline
\end{tabular}

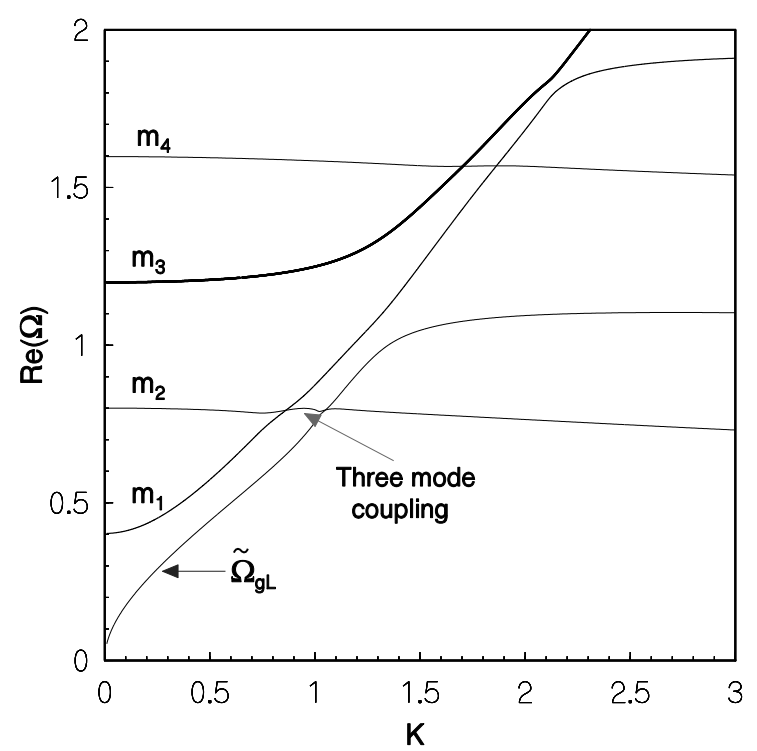

Fig. 5. Region in the diagnostic diagram for moderate field strength $(\epsilon=0.1)$ and $\tilde{\tau}_{\mathrm{R}}=0.5$, where the modified Lamb mode, magnetic modes and the gravity-Lamb mode are present. These results are for zero gradient boundary conditions.
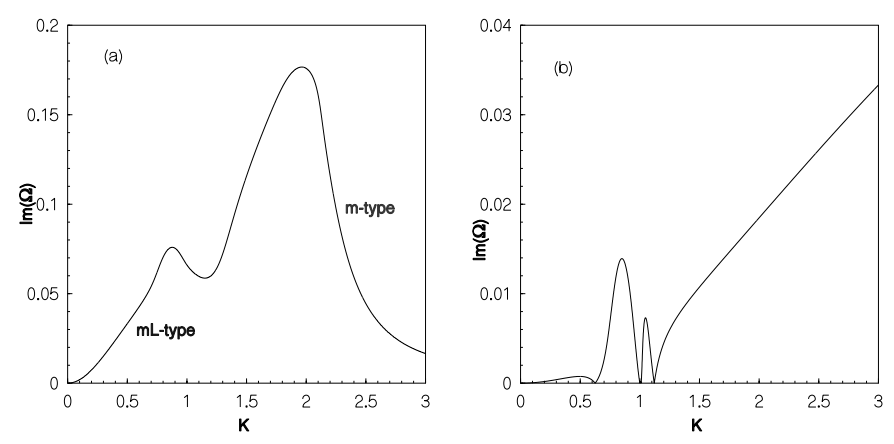

Fig. 6. Variation of the imaginary part of $\Omega$ with $K$ for the same set of parameters as in Fig. 5. Panel a) shows the $m_{1}$-mode and panel b) the $m_{2}$-mode.

variation of the imaginary part of the frequency for the $m_{2}-$ mode (Fig. 5) with $K$. The two peaks correspond to modified Lamb and $m$-mode coupling and modified gravity-Lamb $(g L-)$ and $m$-mode coupling respectively. Note the steep rise of the imaginary part after the avoided crossing which indicates the effect of the gravity mode. Figure 5 also shows the lower branch of the modified gravity-Lamb mode (indicated as $\tilde{\Omega}_{g L}$ ) which was absent in the purely adiabatic case.
Table 2. Eigenfrequencies (corresponding to a sunspot with radius $5000 \mathrm{~km}$ ) of different $p$-modes for a model atmosphere with $D=20$, $B=2 \mathrm{kG}, \tilde{\tau}_{\mathrm{R}}=0.5$.

\begin{tabular}{ccccccc}
\hline \hline Mode & $\operatorname{Re}(\Omega)$ & $\operatorname{Im}(\Omega)$ & $P(\mathrm{~S})$ & $v(\mathrm{mHz})$ & $\tau_{D}(\mathrm{~S})$ & $\tau_{D} / P$ \\
\hline$p_{1}$ & 0.520 & 0.0659 & 186 & 5.4 & 233 & 1.25 \\
$p_{2}$ & 0.605 & 0.0767 & 160 & 6.2 & 200 & 1.25 \\
$p_{3}$ & 0.706 & 0.089 & 137 & 7.3 & 173 & 1.26 \\
$p_{4}$ & 0.820 & 0.104 & 118 & 8.5 & 148 & 1.25 \\
\hline
\end{tabular}

\section{High magnetic field case}

We now consider a situation which is more realistic as far as the solar atmosphere is concerned. We consider an isothermal atmosphere extending over several scale heights for which $v_{\mathrm{A}} \gg c_{\mathrm{S}}$ over most of the atmosphere. This situation is somewhat similar to the atmosphere in sunspots. We consider the solution for small $K$. There are three types of wave modes present in this situation, the slow, fast and magneto-gravity-Lamb $(M g L)$ modes. The $g L-$ mode acquires a more pronounced magnetic behavior because of the higher magnetic field strength and so we call them $M g L$-mode (see Banerjee et al. 1995 for further details). From a study of the energy density variation of these modes we find that the fast and $M g L$-modes are essentially confined to photospheric regions (i.e. the lower part of the atmosphere), whereas the wave energy density of the slow waves is spread over the entire extension of the cavity. As far as the wave heating is concerned the slow modes appear to be a more promising candidate than the other two type of modes.

The frequencies of the slow magneto-acoustic modes or $p$ modes can be found from Eq. (26) with $K=0$, i.e.

$\Omega_{p, n}=\sqrt{\tilde{\gamma}\left(\frac{n^{2} \pi^{2}}{D^{2}}+\frac{1}{4}\right)}$,

where $n$ denotes the order. Following Scheuer \& Thomas (1981) let us treat the sunspot umbra as a cylinder of radius $a$. It can easily be shown that our analysis for a plane can be carried over in a straightforward way to cylindrical geometry, by treating axisymmetric modes and regarding $\xi_{x}$ and $k$ as the radial displacement and wave number respectively. Assuming that the radial component of the displacement vanishes at $r=a$, we find that $k$ takes discrete values given by $k a=j_{1, v}$, where $j_{1, v}$ denotes the zero of the Bessel function $J_{1}$ of order $v$. We consider the lowest-order mode (where order refers to the horizontal direction) corresponding to $v=1$. This provides us with a relation between the horizontal wave number and the radius of the spot. Table 1 represents the eigenfrequencies of different order 


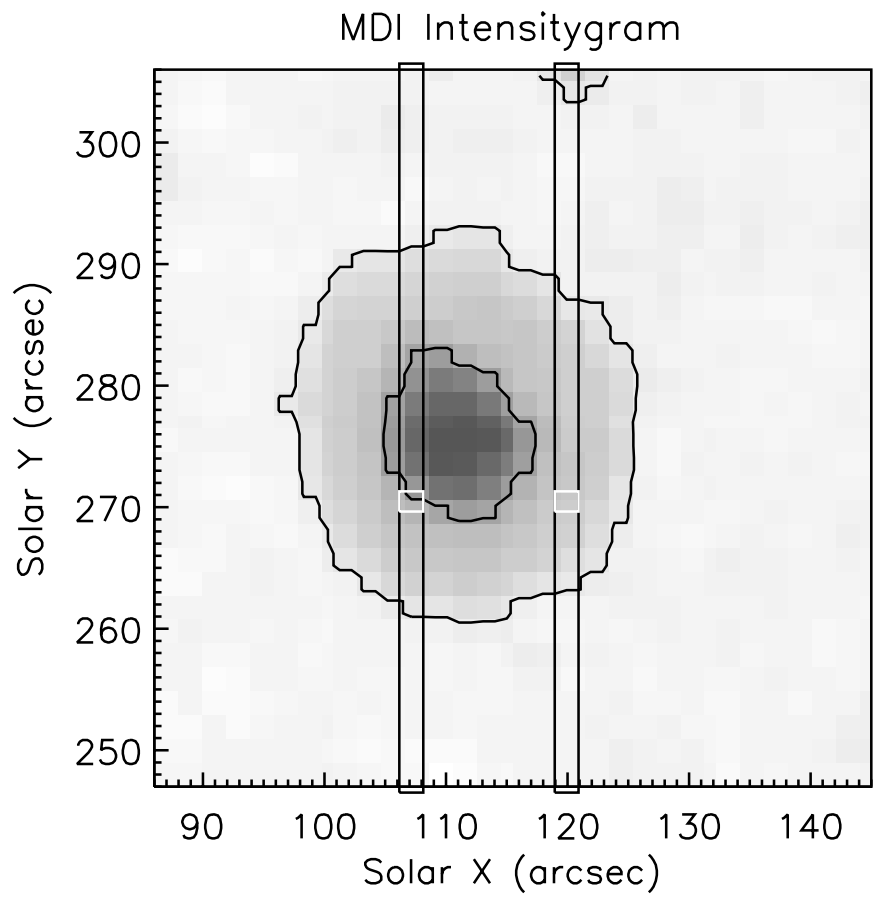

Fig. 7. MDI intensity-gram showing the location of the slit of the s19332r00 dataset, relative to the sunspot umbra and penumbra. The over-plotted black rectangles are the locations of the slit at the start (right) and at the end time (left) of the observations. Pixel number 67 is marked with a white box.

$p$-modes from our model atmosphere with $D=10, \tilde{\tau}_{\mathrm{R}}=0.5$ and $\epsilon=0.84(B \sim 2 \mathrm{KG})$. Table 1 reveals that radiative cooling shifts the eigenfrequencies away from the real axis. Note that the computed frequencies match very well with the ones calculated from expression (32). Radiative cooling leads to a temporal decay of oscillations of the form $\exp \left(-t / \tau_{\mathrm{D}}\right)$. The frequency eigenvalues of the four modes are listed in Table 2 for a typical sunspot with radius of $5000 \mathrm{~km}(K=0.06)$ together with the ratio of characteristic decay time $\tau_{\mathrm{D}}=\operatorname{Im}(\omega)^{-1}$ and oscillation period $=2 \pi / \operatorname{Re}(\omega)$. In the presence of Newtonian cooling all four modes are damped by a factor $\mathrm{e}^{-1}$ within two oscillation periods.

\section{Observations and data reduction}

For these observations we have used the normal incidence spectrometer (NIS) (Harrison et al. 1995), which is one of the components of the Coronal Diagnostic Spectrometer (CDS) on-board the Solar Heliospheric Observatory (SoHO). The data discussed here were selected from the observing period 14 April and 19-20 April 2000. The observations were performed for two different active regions. The details of the observations including pointing and start times are summarized in Table 3. Two different cDs sequences were run, one temporal series sequence called CHROM_N6 and another raster sequence called CHROM_N5. Temporal series datasets of $\sim 85$ min duration were obtained for the three lines of He I $584 \AA(\log T=4.6)$, O III $599 \AA(\log T=5.0)$ and O v $629 \AA(\log T=5.4)$ using exposure times of $25 \mathrm{~s}$ and the $2 \times 240 \operatorname{arcsec}^{2}$ slit. The cDs pixels in the $y$ direction (i.e. spatial resolution) are of size
1.68 arcsec. In the raster sequence the $2 \times 240$ slit was moved 30 times in steps of $2 \operatorname{arcsec}$ so as to build up $60 \times 240 \operatorname{arcsec}^{2}$ raster images within a duration of $24 \mathrm{~min}$. For this sequence the lines used were: He I $584 \AA(\log T=4.6)$, O III $599 \AA(\log T=$ 5.0), O v $629 \AA(\log T=5.4), \mathrm{Ca} \times 574 \AA(\log T=5.8)$, and Mg Ix $368 \AA(\log T=6.0)$.

In order to get good time resolution the rotational compensation was switched off (sit-and-stare mode) and so it becomes important to calculate the lowest possible frequency we can detect from this long time sequence after taking the solar rotation into account (see Doyle et al. 1998 for details). We estimate that the maximum effect of the sit and stare mode on the resulting power, for all datasets would be a spreading of the frequencies by around $1.5 \mathrm{mHz}$, depending on the size of the source. For all our sunspots, whose sizes are several arcsec and which have (well-defined) primary oscillating frequencies much above $3 \mathrm{mHz}$, the effect of the sit and stare is not considered to be important.

The fitting of the different CDS lines was done using a single Gaussian as the lines were found to be generally symmetric. Details on the cDs reduction procedure, plus the wavelet analysis, may be found in O'Shea et al. (2001). Before applying the wavelet analysis we first removed the trend of the data (i.e. the very lowest frequency oscillations) using a 30 point running average. By dividing the results of this running average (or trend) into the original data and subtracting a value of one we obtained the resulting detrended data used in the analysis. Fludra (2001) have shown that this method is very efficient in removing the low frequency background oscillation. The statistical significance of the observed oscillations was estimated using a Monte Carlo or randomization method. The advantage of using a randomization test is that it is distribution free or nonparametric, i.e. it is not limited or constrained by any specific noise models, such as Poisson, Gaussian etc. We follow the method of Fisher randomization as outlined in Nemec \& Nemec (1985), performing 250 random permutations to calculate the probability levels. The levels displayed here are the values of $(1-p) \times 100$, where $p$ is the proportion of the permutations that show a null test result (see O'Shea et al. 2001). We choose a value of $95 \%$ as the lowest acceptable probability level. Occasionally the estimated $p$ value can have a value of zero, i.e. there being an almost zero chance that the observed time series oscillations could have occurred by chance. In this case, and following Nemec \& Nemec (1985), the 95\% confidence interval can be obtained using the binomial distribution, and is given by $0.0<p<0.01$, that is, the probability $((1-p) \times 100)$ in this case is between $99-100 \%$. The velocity values presented in this paper are relative velocities, that is, they are calculated relative to an averaged profile, obtained by summing over all pixels along the slit and all time frames.

\section{Observational results}

We first present results from the sunspot in active region 8951 as observed on 14 April 2000. In Fig. 7, using an MDI intensity-gram image, we show an enlarged region around the sunspot together with an overlay of a portion of the slit from the temporal series dataset s19932r00 showing its 

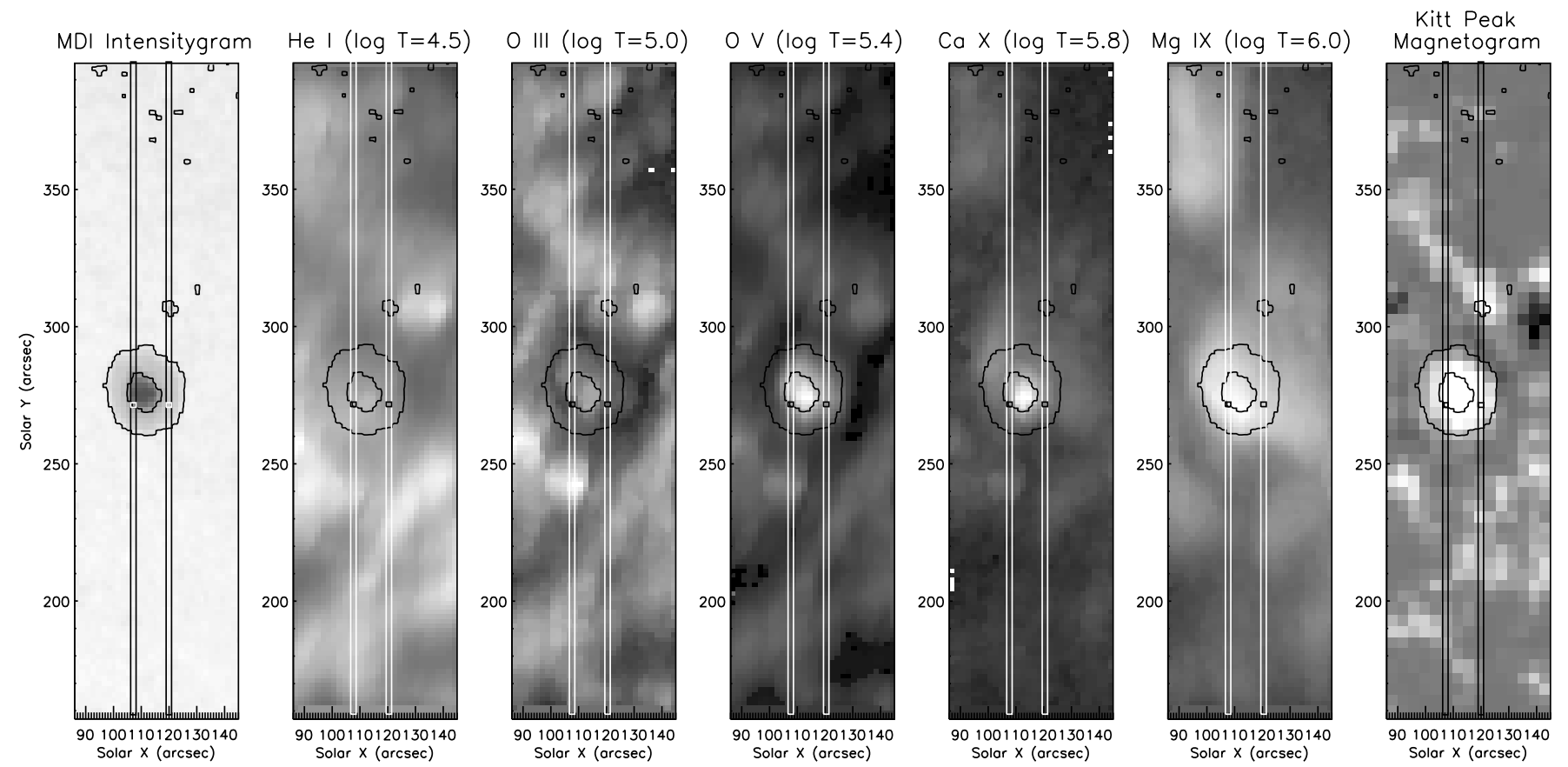

Fig. 8. cDs raster images for the s19331r00 dataset, in different temperature lines (as labeled), and the Kitt peak magnetogram. The over-plotted white rectangles are the locations of the slit for the s19332r00 dataset at the start (right) and the end time (left). Pixel 67 is marked as a black box on the images. The contours indicate the location of the umbra and penumbra.

Table 3. A log of the datasets used in this paper obtained during April 2000.

\begin{tabular}{|c|c|c|c|c|c|c|}
\hline $\begin{array}{l}\text { Active } \\
\text { region }\end{array}$ & Date & Dataset & $\begin{array}{l}\text { Type of } \\
\text { observation }\end{array}$ & $\begin{array}{l}\text { Pointing } \\
(X, Y)\end{array}$ & $\begin{array}{l}\text { Starting time } \\
\text { UT }\end{array}$ & "Lines used \\
\hline \multirow[t]{6}{*}{ AR 8951} & 14 April 2000 & s19331r00 & Raster & $(116,277)$ & $04: 25$ & $\mathrm{O}_{\text {III, }} \mathrm{O}_{\mathrm{v}, \mathrm{He}} \mathrm{I}, \mathrm{Mg}_{\mathrm{IX}}, \mathrm{Ca} \mathrm{x}$ \\
\hline & & s19332r00 & Temporal & $(122,277)$ & $04: 48$ & $\mathrm{O}_{\text {III, }} \mathrm{O}_{\mathrm{v}, \mathrm{He}_{\mathrm{I}}}$ \\
\hline & & s19333r00 & Raster & $(135,277)$ & $06: 14$ & $\mathrm{O}_{\text {III }}, \mathrm{O}_{\mathrm{v}}, \mathrm{He}_{\mathrm{I}}, \mathrm{Mg}$ Ix, $\mathrm{Ca} \mathrm{x}$ \\
\hline & & s19334r00 & Temporal & $(136,277)$ & $06: 37$ & $\mathrm{O}_{\text {III, }} \mathrm{O}_{\mathrm{v}, \mathrm{He}_{\mathrm{I}}}$ \\
\hline & & s19335r00 & Raster & $(152,276)$ & 08:02 & $\mathrm{O}_{\text {III }}, \mathrm{O}_{\mathrm{v}, \mathrm{He}}, \mathrm{Mg}$ Ix, $\mathrm{Ca} \mathrm{x}$ \\
\hline & & s19336r00 & Temporal & $(153,275)$ & $08: 26$ & $\mathrm{O}_{\text {III, }} \mathrm{O}$ v, $\mathrm{He}_{\mathrm{I}}$ \\
\hline \multirow[t]{6}{*}{ AR 8963} & 19 April 2000 & s19377r00 & Raster & $(-1,418)$ & $18: 21$ & $\mathrm{O}_{\mathrm{III}}, \mathrm{O}$ v, $\mathrm{He}_{\mathrm{I}}, \mathrm{Mg}$ Ix, $\mathrm{Ca}$ x \\
\hline & & s19378r00 & Temporal & $(4,412)$ & $18: 45$ & $\mathrm{O}$ III, O v, He I \\
\hline & & s19379r00 & Raster & $(18,416)$ & $20: 10$ & $\mathrm{O}_{\text {III, }} \mathrm{O}$ v, $\mathrm{He}$ I, $\mathrm{Mg}$ Ix, $\mathrm{Ca}$ x \\
\hline & & s19380r00 & Temporal & $(20,415)$ & $20: 34$ & $\mathrm{O}_{\text {III, }} \mathrm{O}_{\mathrm{v}, \mathrm{He}_{\mathrm{I}}}$ \\
\hline & & s19381r00 & Raster & $(33,416)$ & $21: 59$ & $\mathrm{O}_{\text {III, }} \mathrm{O}_{\mathrm{v}, \mathrm{He}}, \mathrm{Mg}$ Ix, $\mathrm{Ca}$ x \\
\hline & & s19382r00 & Temporal & $(32,414)$ & $22: 23$ & $\mathrm{O}_{\text {III, }} \mathrm{O}_{\mathrm{v}, \mathrm{He}_{\mathrm{I}}}$ \\
\hline \multirow[t]{6}{*}{ AR 8963} & 20 April 2000 & s19387r00 & Raster & $(204,402)$ & $18: 00$ & O III, O v, He I, Mg Ix, Ca x \\
\hline & & s19388r00 & Temporal & $(204,402)$ & $18: 24$ & $\mathrm{O}_{\mathrm{III}}, \mathrm{O}_{\mathrm{v}, \mathrm{He}_{\mathrm{I}}}$ \\
\hline & & s19389r00 & Raster & $(217,401)$ & $19: 49$ & O III, O v, He I, Mg Ix, Ca x \\
\hline & & s19390r00 & Temporal & $(218,401)$ & $20: 13$ & $\mathrm{O}_{\text {III }}, \mathrm{O}_{\mathrm{v}, \mathrm{He}_{\mathrm{I}}}$ \\
\hline & & s19391r00 & Raster & $(232,401)$ & $21: 38$ & $\mathrm{O}_{\text {III }}, \mathrm{O}_{\mathrm{v}}, \mathrm{He}_{\mathrm{I}}, \mathrm{Mg}$ Ix, $\mathrm{Ca}$ x \\
\hline & & s19392r00 & Temporal & $(233,401)$ & $22: 02$ & $\mathrm{O}_{\mathrm{III}}, \mathrm{O} \mathrm{v}, \mathrm{He}_{\mathrm{I}}$ \\
\hline
\end{tabular}

location at the beginning and end times of the observation. The MDI intensity-gram used was obtained from the file fd_Ic_01h.63844.0048.fits. The MDI intensity-gram observations were performed at 04:47:33, the same time approximately as the start time of the CDS temporal series observations of dataset s19332r00. In Fig. 8 we show cDs intensity rasters of size $60 \times 240 \operatorname{arcsec}^{2}$ for different temperature lines along with a (low resolution) Kitt peak magnetogram. The cDs rasters were obtained from dataset s19331r00, with a starting time of 04:25 on the 14 April 2000. The low resolution Kitt Peak magnetogram (resolution $\sim 4$ arcsec) with a start-time of $14: 26: 31$ on the same day was thus obtained about 10 hours after the CDS observations. The thin rectangles over-plotted on these images show the location of the slit (for the s19332r00 dataset) at the beginning (the right one) and end of the temporal sequence (the left one). Pixel 67 is marked as a black box in all the images. The CDS rasters shown are the square-root images (i.e. the square root of the intensities has been taken to reduce the contrast between the most bright and dark values). A comparison of the raster images and the magnetogram clearly 

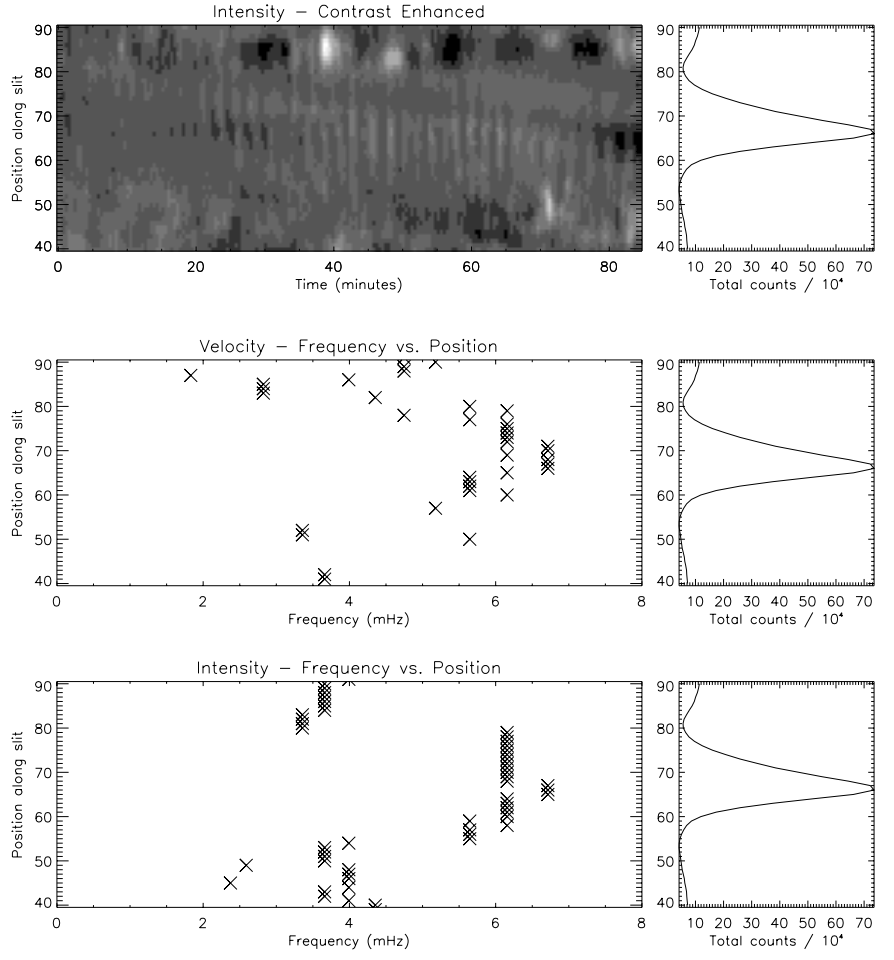

Fig. 9. Frequencies measured as a function of spatial position along the slit (X-F slice) for the O v $629 \AA$ line (left panels) and the s19332r00 dataset. The right panels show the total number of counts in a pixel (summed counts) over the observation time.

reveals that all the intensity enhancements are closely related with concentrated magnetic field regions.

The contours for the umbra and penumbra (in Figs. 7, 8) are plotted using the average value for the whole MDI intensitygram as a guide. The penumbra is defined as the parts of the MDI intensity where the intensity falls below a factor of 1.5 that of the average, i.e. it is the average/1.5. The outer contour around the sunspot shows the contour of the average value, while the inner contour shows the contour of the average/ 1.5 values. The umbra is then defined as anything that is contained within this average/ 1.5 contour.

In order to show the spatial variation of the observed oscillation frequencies across the sunspot region we select the strongest line, i.e. O v $629 \AA$. In Fig. 9 we plot the variation of the frequencies over a section of the observing slit. This section includes the umbra, penumbra and the adjacent regions. The top panel shows a contrast enhanced intensity map (X-T slice), obtained by removing the low frequency trend of the oscillations for each of the positions along the slit. The lower two left panels show the measured frequencies as a function of position along the slit (X-F slice) for velocity and intensity respectively. The crosses correspond to the primary maxima in the global wavelet spectra. The total number of counts in a pixel (summed counts) during the observation is shown in the right columns and is useful in identifying the location of the umbra of the sunspot. Note that pixel 67 is the brightest pixel across our slit (see right panel of Fig. 9). This pixel may correspond to a plume region. According to Maltby et al. (1999), locations that show $I>5 \bar{I}$, where $\bar{I}$ is the average intensity in the sunspot area being investigated, can be considered as plumes in the sunspot umbra, if they also coincide with the location of an umbral region seen in, for example, a MDI intensity-gram. In the X-T slice, for portions of the image, roughly from 50-60 and then between 80-90 pixels along the slit there are brightenings and darkenings, representing longer period oscillations, which correspond to the penumbra and adjacent regions. The frequencies in these regions are typically in the $2-4 \mathrm{mHz}$ range, whereas for the central part of the slit, roughly from pixels 60-75, there are many alternate dark and bright ridges, corresponding to the umbral oscillations, with frequencies of oscillation in the range 5.5-7 mHz, with most peaks at $6.2 \mathrm{mHz}$. Another point to note in the X-T image is that there is a drift in the ridges, a slanting from top to bottom, which is due to the solar rotational drift (i.e. a sit and stare effect), that is, the oscillating umbra source moves down along the slit as the Sun rotates under the slit. As mentioned before, the peak counts occur at pixel 67. Below we investigate this pixel in all three spectral lines, He I, O III and $\mathrm{Ov}$.

In Fig. 10 we show as a representative umbral oscillation, the power spectra analysis corresponding to the He I $584 \AA$ line at pixel location 67 (marked as a box in Fig. 8). In the wavelet spectrum, the dark contour regions show the locations of the highest powers. Only locations that have a probability greater than $95 \%$ are regarded as being real, i.e. not due to noise. Crosshatched regions, on either side of the wavelet spectrum, indicate the "cone of influence" (COI), where edge effects become important (see Torrence \& Compo 1998). The dashed horizontal lines in the wavelet spectra indicate the lower frequency cut-off, in this instance $1.5 \mathrm{mHz}$. The results from the phase plots show that the $\mathrm{He}$ I $584 \AA$ intensity and velocity both show significant power in the $6.0-7.0 \mathrm{mHz}$ range, for the periods between the 20-30th and 40-65th minutes of the observing sequence. From the overlay of the MDI intensity-gram and the slit location (Fig. 7) one can clearly see that this particular pixel was over the sunspot umbra between the time interval 2065 th minute of the observing sequence (for a total of $45 \mathrm{~min}$ ). We should point out that the wavelet analysis has been carried out on relative intensity and velocity values and hence there is a lack of low frequency power in the wavelet spectrum plots. The global wavelet spectra (on the right of Figs. 10a, b, which are the average of the wavelet power spectrum over the entire observing period, show the strongest intensity and velocity power at $6.2 \mathrm{mHz}(\sim 161 \mathrm{~s})$. This is printed out in Fig. 10 above the global wavelet plots, together with the probability estimate for the global wavelet power spectrum. In the lowest panels we show the variation of the probability level as estimated from the randomization test. Note that the statistical significance is calculated only for the maximum powers in the wavelet spectrum marked by the dotted white line in the dark patches. From these panels we can clearly see that the oscillations were significant in the period between the $20-30$ and 40-65 min of the time sequence.

The O III $599 \AA$ line formed in the low-to-mid transition region, is rather faint and to increase the signal to noise ratio we binned over two pixels (67-68). The intensity wavelet shows power around $5.6 \mathrm{mHz}$ and also some strong power around $3 \mathrm{mHz}$ for the first $20 \mathrm{~min}$. The slit was not positioned over 


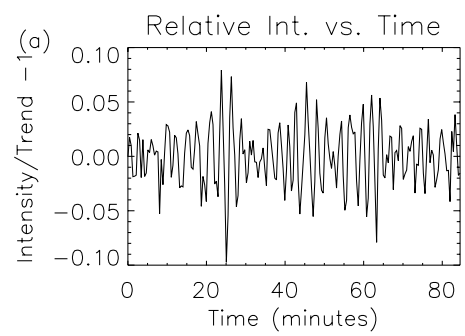

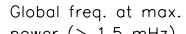
power $(>1.5 \mathrm{mHz})$ $=6.2 \mathrm{mHz}$ Prob. level: $99-100 \%$ Intensity Wavelet
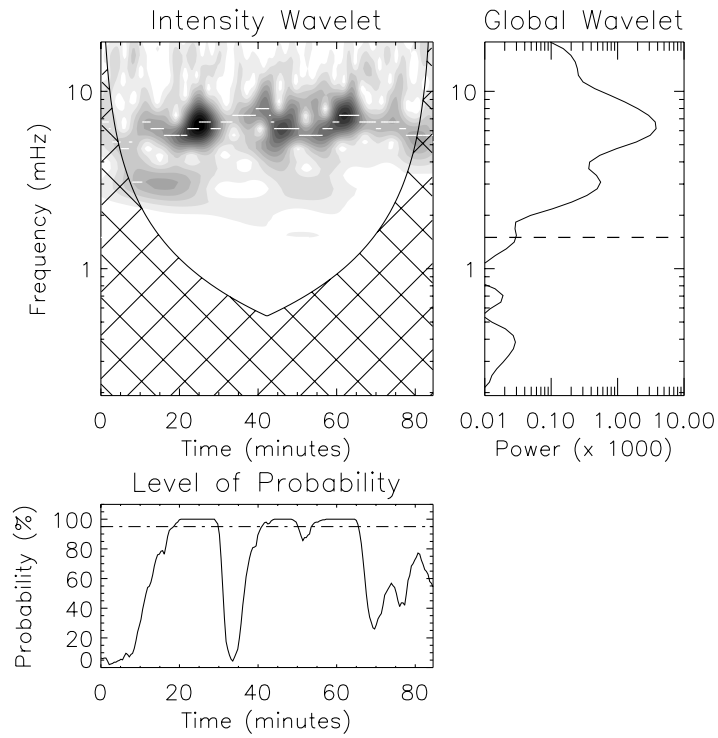

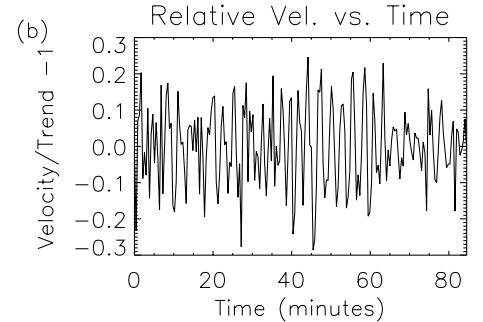

Velocity Wavelet
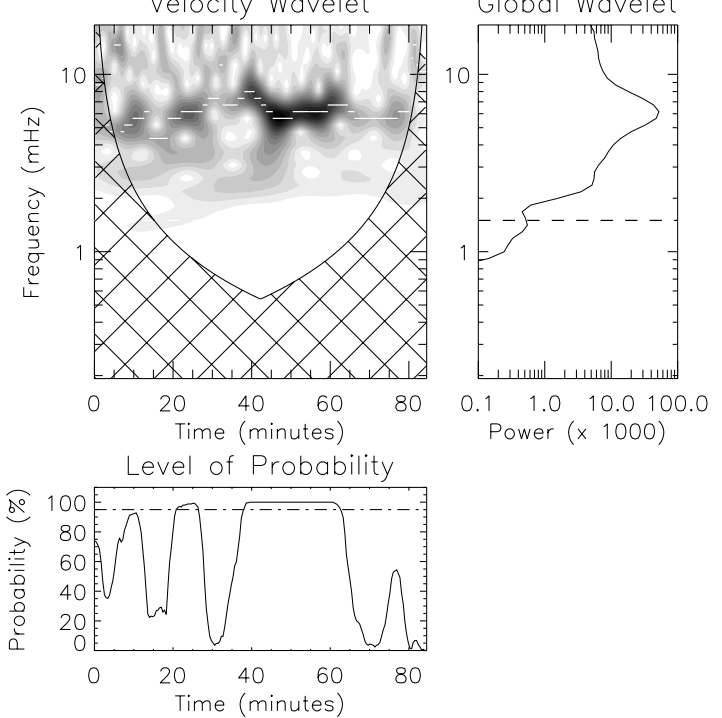

Global freq. at max. power $(>1.5 \mathrm{mHz})$ $=6.2 \mathrm{mHz}$ Prob. level: $99-100 \%$

Fig. 10. Wavelet results corresponding to the He $584 \AA$ line in the s $19332 \mathrm{r} 00$ dataset at pixel 67 . Panels a) and b) represent intensity and velocity results respectively. The middle row left panels show the time frequency phase plot corresponding to the variations shown in the top panels. The middle row right hand panels show the average of the wavelet power spectrum over time, i.e. the global wavelet spectrum. The continuous dashed horizontal lines in the wavelet spectra indicate the lower cut off frequency. The lowest panels show the variation of the probability with time from the randomization test, with the dot-dash line indicating the $95 \%$ significance level.

the sunspot umbra for the first 20 min of the observing sequence and thus the first $20 \mathrm{~min}$ power corresponds to the umbra boundary. In the global wavelet the main power peak is at $5.6 \mathrm{mHz}$, but there is also a strong peak around $3 \mathrm{mHz}$, which corresponds to the first $20 \mathrm{~min}$ during which the penumbra rotates under the slit. The most significant oscillations take place during the time intervals between $20-25$ and $45-50$ min. For the $\mathrm{O}$ III $599 \AA$ Aine the velocity signal is too weak and hence the oscillations for this component are not reliable and so are not included in this analysis.

Now we turn our attention to the transition region O v $629 \AA$ line. Figure 11 shows the wavelet results for the same pixel location, 67, and dataset, s19332r00. Intensity and velocity both shows strong power around $6.7 \mathrm{mHz}$ in the phase and global wavelet spectra. The $\mathrm{O} v$ oscillation is strong for the same time interval (as in $\mathrm{He}_{\mathrm{I}}$ and $\mathrm{O}$ III), namely between the 20-65 min, with a drop in significance for the time interval between 30-40 min of the sequence. From this one pixel located in the sunspot umbra we thus find that the average frequency of oscillation over the entire observing time for the $\mathrm{O} v$ line is at $6.7 \mathrm{mHz}(165 \mathrm{~s})$, as estimated from the global wavelet spectrum.

Using the same techniques as before, we also examine the same sunspot $\sim 30 \mathrm{~min}$ later using dataset s19334r00. The results from this temporal series dataset are summarized in
Table 4. For the central portion of the umbra we find that the global peaks show frequencies in the range $5.2-5.7 \mathrm{mHz}$ for all the three lines observed and the lifetimes of the oscillations are between 10-20 min, very similar to the previous case of s19332r00. We concentrate on the same single pixel as before, namely pixel 67 , in the umbra of the sunspot (plume). From Fig. 12 it is clear that the main oscillations in intensity and velocity, take place in a 5-60 min interval in the observing sequence, with most significant oscillations occurring in the 5-15 and 25-65 min intervals for intensity and the 20-55 min interval for the velocity (this can be confirmed by looking at the variation of probability in the lowest panels). The global peak for the intensity and velocity both appear at $6.2 \mathrm{mHz}$ with a very high probability level. The results from the other lines from this dataset are given in Table 4. In Fig. 13, we show the overall spatial variations of the oscillations in $\mathrm{O} v$. In the $\mathrm{X}$-T slice we can see faint light and dark ridges in the interval between pixels $60-75$, where the central part, roughly between pixels 66-68 corresponds to the plume (where $I>5 \bar{I}$, see rightmost panels). Once again a slow downward drift of the bright ridges may be seen in the X-T slice, which is a rotational effect due to the movement of the oscillating source down relative to the slit with time. The frequency distribution shows that the umbra oscillates in intensity and velocity in the $5-6.5 \mathrm{mHz}$ range, with most oscillations occurring at $6.2 \mathrm{mHz}$. 

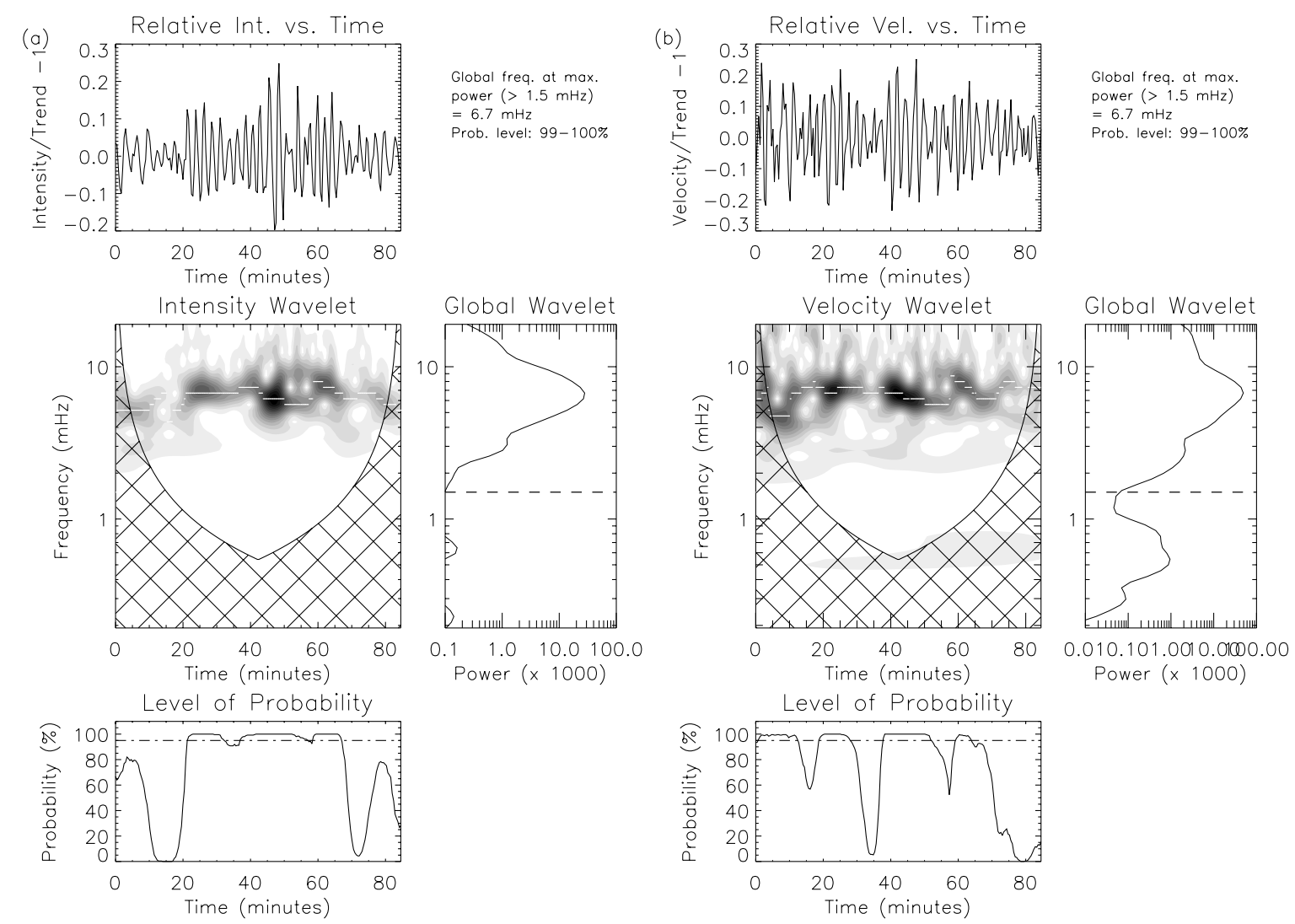

Fig. 11. Wavelet results corresponding to the O v $629 \AA$ line in the s 19332 r00 dataset at pixel 67 . Representations are same as Fig. 10.

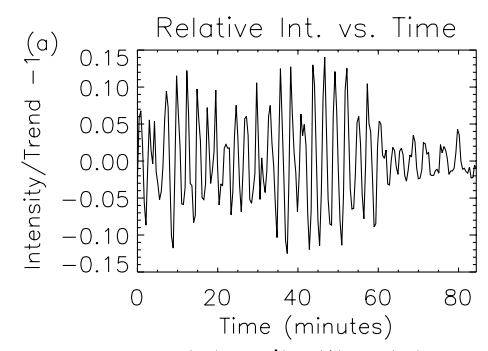

Global freq. at max power $(>1.5 \mathrm{~m}$
$=6.2 \mathrm{mHz}$

Prob. level: $99-100 \%$
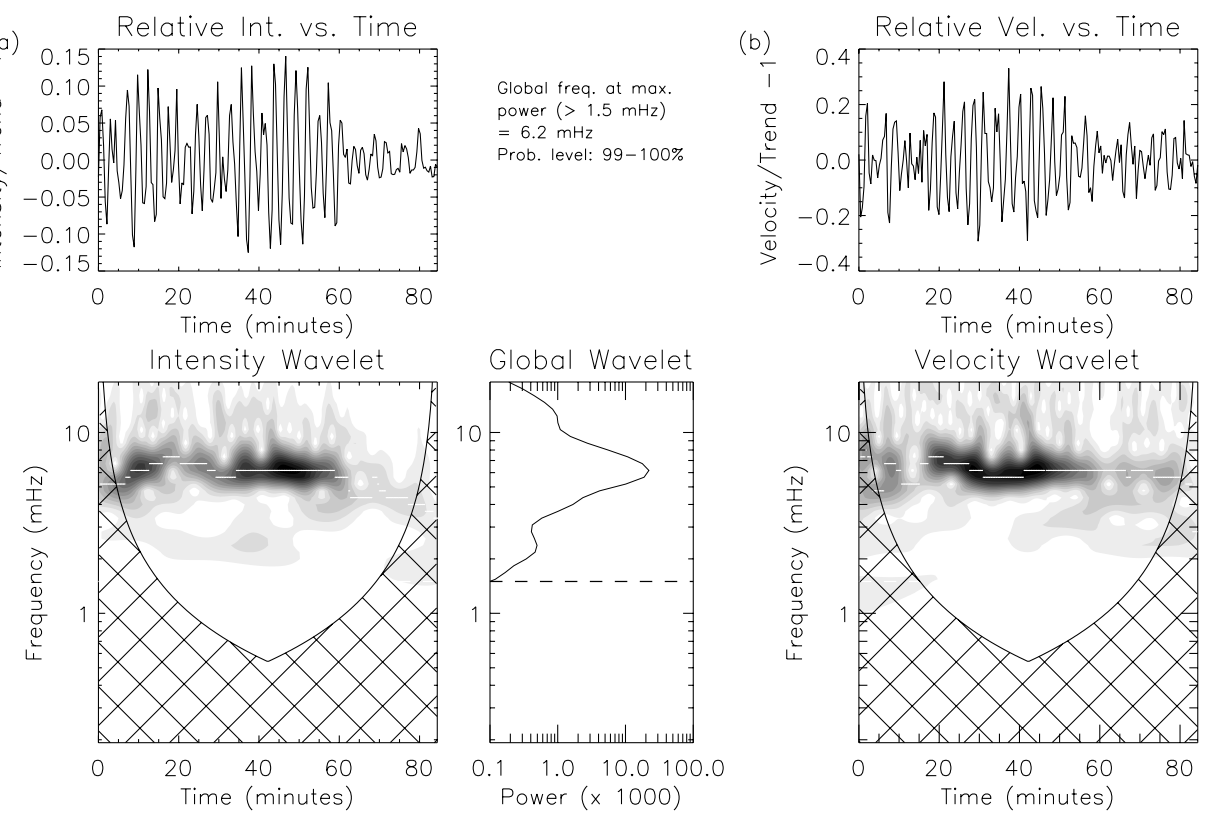

Global freq. ot max. power $(>1.5 \mathrm{mH}$
$=6.2 \mathrm{mHz}$

Prob. level: $99-100 \%$
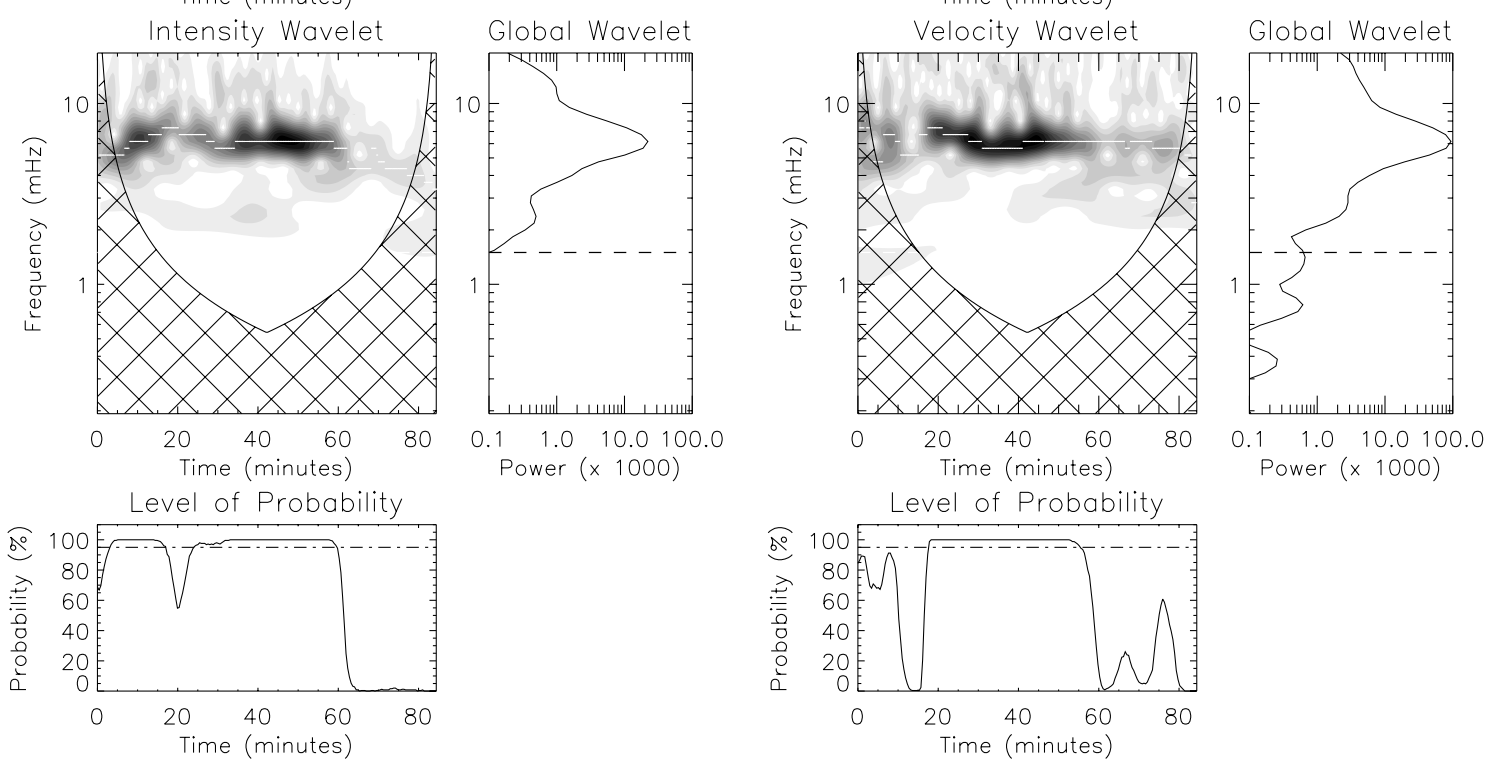

Fig. 12. Wavelet results corresponding to the O v 629 A line in the s $19336 \mathrm{r} 00$ dataset at pixel 67. Representations are the same as Fig. 10. 
Now we turn our attention to the study of the other active region, AR8963 over the period 19-20 April 2000 (see Table 3 for details). To save space, we do not show here detailed wavelet plots for individual dataset, rather we choose some selective pixel locations in each dataset (corresponding to the umbra of a sunspot) and summarize our results in the form of Table 4. We also list the duration of the oscillations, estimated as the periods of time different oscillation packets showed significant oscillations above the $95 \%$ significance level. This can be easily measured from a comparison of the wavelet phase plots and the variation of the probability level in the wavelet analysis (e.g. in Fig. 12). We just point out here some of the other additional features which we noticed for this active region. Firstly we should point out that this active region was much larger compared to the previous one with 12 beta type spots. In certain cases we found that the oscillation frequency changed during the observation which might indicate that a new oscillating region was rotating into the field of view of the cDs slit. For 20 April 2000 dataset, we also encountered a small flaring event which interfered with the measurement of the underlying higher frequency oscillations. It was noted that active region AR8963 had slightly evolved in comparison with the previous day (the flare was a result of that). In all the datasets corresponding to 20th April we find a wider distribution of frequency measured from the peak of the global wavelet spectrum, so we have listed the range of frequency over which the oscillation was most significant.

\section{Discussions}

Using UVSP data obtained in emission lines formed at temperature of $7 \times 10^{4} \mathrm{~K}$ to $1.3 \times 10^{5} \mathrm{~K}$, Gurman et al. (1982) observed transition region oscillations in sunspots with frequencies in the range of $5.8-7.8 \mathrm{mHz}$. Their in-phase intensity and velocity oscillations lead them to interpret the oscillations in terms of upward propagating acoustic waves. For the first time Thomas et al. (1987) made simultaneous detection of umbral oscillation at different heights, starting from the chromosphere to the transition region. Their power spectra of intensity and velocity both show multiple peaks at the 3 min band.

With the launch of SoHO there has been renewed interest in the study of umbral oscillations. Fludra $(1999,2001)$ investigated 3 min intensity oscillations with CDs by observing the chromospheric line $\mathrm{He}$ I and several transition region lines. He concluded that the 3 min umbral oscillations can occur both in the so called sunspot plumes (bright features seen in the transition region above sunspot) or in the lower intensity plasma closely adjacent to the plumes. He found the spectral power to be contained in the $5.55-6.25 \mathrm{mHz}$ range. No oscillations were detected by him in the Mg Ix $368 \AA$ line, suggesting that the 3 min oscillation does not propagate into the corona. Tziotziou et al. (2002) have presented two-dimensional intensity and Doppler shift images computed at different wavelengths within the Ca II $8542 \AA$ line. Their power spectrum analysis shows a $6 \mathrm{mHz}$ frequency, for the standing umbral oscillations only for the upper half part of the umbra. For the penumbra they report a $3 \mathrm{mHz}$ frequency. They also conclude that the umbral oscillations are a localized phenomena. SUMER observations (in both
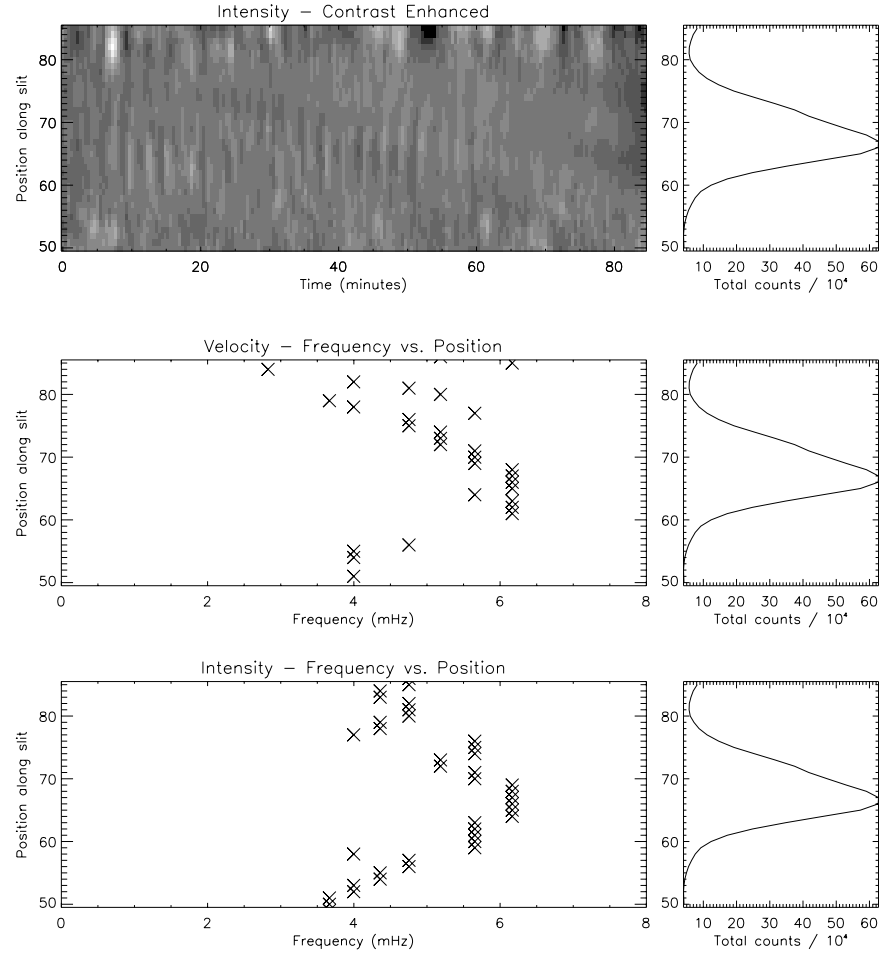

Fig. 13. Frequencies measured as a function of spatial position along the slit (X-F slice) for the $\mathrm{O} v 629 \AA$ line (left panels) and the s19336r00 dataset.

intensity and velocity) have confirmed that the sunspot oscillations are prominent in transition region lines above the umbra (Maltby et al. 2001). They also state that the umbral oscillations are a localized phenomenon and that the 3 min oscillations fill the sunspot umbra in the transition region and tends to stop at the umbral rim. Support for the acoustic wave hypothesis was presented by Brynildsen et al. (1999a,b). They observed oscillations in intensity and velocity to test the hypothesis and found the oscillations to be compatible with upwardly propagating waves.

More recently O'Shea et al. (2002) and Brynildsen et al. (2002) have both presented joint observations of the $3 \mathrm{~min}$ umbral oscillations with TRACE and CDS. O'Shea et al. (2002) find oscillations at all temperatures from the temperature minimum, as observed by TRACE $1700 \AA$ up to the upper corona, as measured by the Fe xvi $335 \AA$ line with cDs. Both these authors report that the oscillation amplitude above the umbra increases with increasing temperature, reaches a maximum in the transition region and decreases for higher temperature lines, though O'Shea et al. (2002) finds evidence for another increase in amplitude for lines formed above $1 \mathrm{MK}$. O'Shea et al. interpreted their observations in terms of slow magneto-acoustic waves propagating upwards (as confirmed from their time delays) along magnetic field lines. In a recent theoretical paper, Zhukov (2002) calculated the spectrum of eigenmodes of umbral oscillations. It was shown that the 3 min umbral oscillations are the $p$-modes modified by the magnetic field.

The salient feature of our observation is that we have detected both intensity and velocity oscillations in chromospheric 
Table 4. Summary of the oscillation frequencies observed corresponding to the different dataset.

\begin{tabular}{|c|c|c|c|c|c|c|c|c|}
\hline \multirow[t]{2}{*}{ Dataset } & \multirow[t]{2}{*}{ Lines } & \multirow[t]{2}{*}{ Pixel } & \multicolumn{3}{|c|}{ Intensity results } & \multicolumn{3}{|c|}{ Velocity results } \\
\hline & & & Freq. maxima & Prob. level & Duration & Freq. maxima & Prob. level & Duration \\
\hline \multirow[t]{3}{*}{ s19332r00 } & $\mathrm{He}_{\mathrm{I}}$ & 67 & $6.2 \mathrm{mHz}$ & $99-100 \%$ & $20-30,40-65$ & $6.2 \mathrm{mHz}$ & $99-100 \%$ & $20-25,40-65$ \\
\hline & $\mathrm{O}_{\text {III }}$ & $67-68$ & $5.6 \mathrm{mHz}$ & $99.2 \%$ & $20-25,45-50$ & & & \\
\hline & $\mathrm{O} v$ & 67 & $6.7 \mathrm{mHz}$ & $99-100 \%$ & $20-30,40-65$ & $6.7 \mathrm{mHz}$ & $99-100 \%$ & $20-30,40-50,60-65$ \\
\hline \multirow[t]{3}{*}{ s19334r00 } & $\mathrm{He}_{\mathrm{I}}$ & 66 & $5.7 \mathrm{mHz}$ & $99-100 \%$ & $10-30,40-50$ & $5.2 \mathrm{mHz}$ & $98.8 \%$ & $15-30,40-50$ \\
\hline & $\mathrm{O}_{\text {III }}$ & $66-67$ & $5.2 \mathrm{mHz}$ & $95.6 \%$ & $15-20,45-55$ & & & \\
\hline & $\mathrm{Ov}$ & 66 & $5.7 \mathrm{mHz}$ & $99-100 \%$ & $15-30,40-55$ & $5.7 \mathrm{mHz}$ & $99-100 \%$ & $15-30,40-50$ \\
\hline \multirow[t]{3}{*}{ s19336r00 } & $\mathrm{He}_{\mathrm{I}}$ & 67 & $6.2 \mathrm{mHz}$ & $99-100 \%$ & $5-10,20-30,40-60$ & & & \\
\hline & $\mathrm{O}_{\text {III }}$ & $66-67$ & $5.7 \mathrm{mHz}$ & $98.4 \%$ & $5-10,40-50$ & & & \\
\hline & $\mathrm{Ov}$ & 67 & $6.2 \mathrm{mHz}$ & $99-100 \%$ & $5-15,25-60$ & $6.2 \mathrm{mHz}$ & $99-100 \%$ & $20-55$ \\
\hline \multirow[t]{3}{*}{ s19378r00 } & $\mathrm{He}_{\mathrm{I}}$ & 20 & $4.8 \mathrm{mHz}$ & $99-100 \%$ & $5-20,25-45$ & & & \\
\hline & $\mathrm{O}_{\text {III }}$ & $21-22$ & $4.8 \mathrm{mHz}$ & $99-100 \%$ & $10-20,25-50$ & $4.8 \mathrm{mHz}$ & $98.8 \%$ & $10-20,40-50$ \\
\hline & $\mathrm{Ov}$ & 21 & $4.8 \mathrm{mHz}$ & $99-100 \%$ & $10-20,25-45$ & $4.8 \mathrm{mHz}$ & $99-100 \%$ & $0-20$ \\
\hline \multirow[t]{3}{*}{ s19380r00 } & $\mathrm{He}_{\mathrm{I}}$ & 21 & $4.8 \mathrm{mHz}$ & $99-100 \%$ & $5-20,25-30,45-55$ & & & \\
\hline & $\mathrm{O}_{\text {III }}$ & $21-22$ & $5.2 \mathrm{mHz}$ & $99-100 \%$ & $5-20,45-55$ & & & \\
\hline & $\mathrm{Ov}$ & 21 & $4.8 \mathrm{mHz}$ & $99-100 \%$ & $5-20,45-55$ & $4.0 \mathrm{mHz}$ & $99.8 \%$ & $5-20$ \\
\hline \multirow[t]{3}{*}{ s19382r00 } & $\mathrm{He}_{\mathrm{I}}$ & 21 & $5.2 \mathrm{mHz}$ & $99-100 \%$ & $45-70$ & & & \\
\hline & $\mathrm{O}$ III & $21-22$ & $5.6 \mathrm{mHz}$ & $99-100 \%$ & $45-60$ & & & \\
\hline & $\mathrm{Ov}$ & 21 & $5.2 \mathrm{mHz}$ & $99-100 \%$ & $10-20,25-35,45-65$ & $4.0 \mathrm{mHz}$ & $99-100 \%$ & $25-35$ \\
\hline \multirow[t]{3}{*}{ s19388r00 } & $\mathrm{He}_{\mathrm{I}}$ & 33 & $3.5-5.0 \mathrm{mHz}$ & $99-100 \%$ & $0-35$ & $3.5-5.0 \mathrm{mHz}$ & $99-100 \%$ & $5-35$ \\
\hline & O III & $32-33$ & $4.0-5.0 \mathrm{mHz}$ & $99-100 \%$ & $0-45$ & $5.7 \mathrm{mHz}$ & $95.0 \%$ & $5-10,35-45$ \\
\hline & $\mathrm{O} v$ & 33 & $4.0-5.0 \mathrm{mHz}$ & $99-100 \%$ & $5-20,35-55$ & $5.2 \mathrm{mHz}$ & $99-100 \%$ & $40-55$ \\
\hline \multirow[t]{3}{*}{ s19390r00 } & $\mathrm{He}_{\mathrm{I}}$ & 33 & $3.5-5.0 \mathrm{mHz}$ & $99-100 \%$ & $5-35$ & $4.5-5.0 \mathrm{mHz}$ & $95.0 \%$ & $0-20$ \\
\hline & $\mathrm{O}_{\text {III }}$ & $32-33$ & $3.5-5.0 \mathrm{mHz}$ & $95.0 \%$ & $5-10,25-30$ & & & \\
\hline & $\mathrm{Ov}$ & 33 & $3.0-5.0 \mathrm{mHz}$ & $99-100 \%$ & $10-45$ & & & \\
\hline \multirow[t]{3}{*}{ s19392r00 } & $\mathrm{He}_{\mathrm{I}}$ & 32 & $3.0-6.0 \mathrm{mHz}$ & $99-100 \%$ & $30-75$ & $4.4 \mathrm{mHz}$ & $99.2 \%$ & $10-20,40-45$ \\
\hline & $\mathrm{O}_{\text {III }}$ & $31-32$ & $4.8 \mathrm{mHz}$ & $98.4 \%$ & $30-40$ & & & \\
\hline & $\mathrm{Ov}$ & 32 & $3.0-5.0 \mathrm{mHz}$ & $99-100 \%$ & $0-10,35-50,65-70$ & $3.5-5.0 \mathrm{mHz}$ & $99-100 \%$ & $0-20,40-45$ \\
\hline
\end{tabular}

and transition region lines as observed by CDS. We should point out that the velocity resolution of $\mathrm{CDs}$ is, at best, $5 \mathrm{~km} \mathrm{~s}^{-1}$, and generally it is quite difficult to detect velocity oscillations with any confidence from noisy data. But with inclusion of a reliable probability test and wavelet technique we were able to extract velocity information in most of the cases with a $95 \%$ confidence level or higher. Most of the earlier work on sunspot oscillations with cDs (Fludra et al.1999, 2001; Brynildsen et al. 2002; O'Shea et al. 2002) presented only intensity results. Our results clearly show that the $3 \mathrm{~min}$ intensity and velocity oscillations are a property of the umbra, and not just the sunspot plume (Figs. 9 and 13, shows that power peak around $6 \mathrm{mHz}$ is present over several pixels in the umbra). We also detect $3 \mathrm{mHz}$ oscillations corresponding to the penumbra, which supports the recent observation by THEMIs, Tziotziou et al. (2002). We should point out that the He I line is thought to have a complex formation history and its emission may not correspond to that which one might expect from a chromospheric line. It is believed that there are two main mechanisms by which $\mathrm{He}$ I can form (Andretta \& Jones 1997); either by collisional excitation in the lower transition region from electrons with kinetic temperature higher than the local temperature of the helium atom or by a process in which coronal photons penetrate into the chromosphere and photoionize helium atoms which then recombine to form $\mathrm{He}$ I. Thus the emission from $\mathrm{He}$ I can reflect conditions at temperatures above that of its putative formation temperature. Furthermore, the definition of where the chromosphere ends and transition region begins is a bit arbitrary. Thus there exists an uncertainty and controversy over the He I formation height. Moreover, the $\mathrm{O}$ III and $\mathrm{O} v$ lines are formed close to each other in temperature. This does not allow us to make a time-delay analysis for the calculation of the wave propagation speed using this data.

\section{Conclusion}

There exists several observational reports of umbral oscillations in the literature and there have been several 
theoretical attempts to explain them. However, no generally accepted model exists for the understanding of these mechanical structures, their physical mechanisms and energy transport to the surroundings. In this paper we presented new solutions for magneto-atmospheric waves in an isothermal atmosphere with a vertical magnetic field in the presence of radiative heat exchange based on Newton's law of cooling. Radiation can radically alter the dynamical properties of wave modes in a fluid. This radiative heat exchange gives rise to a temporal decay of oscillations with a characteristic dimensionless decay time $\tilde{\tau}_{\mathrm{D}}=1 / \Omega_{\mathrm{I}}$, where $\Omega_{\mathrm{I}}$ is the imaginary part of $\Omega$. Depending on the value of the radiative relaxation time $\tilde{\tau}_{R}$, the modes are effectively damped by the radiative dissipation in as short a time as two oscillation periods; however, in the limits of very large or very small $\tilde{\tau}_{\mathrm{R}}$, corresponding to nearly adiabatic or nearly isothermal oscillations, the modes are essentially undamped. We would also like to point out the merits and demerits of using Newton's law to model heat exchange. At sufficiently low frequencies, the wavelength of a disturbance is so long, that it becomes optically thick (no matter how transparent the material is), and the Newtonian cooling approximation no longer holds. Conversely, at high frequencies the wavelength of a disturbance becomes so small that it is optically thin (no matter how opaque the material) and the Newtonian approximation holds good. Bünte \& Bogdan (1994) have already pointed out that radiative effects on oscillations in photospheric and higher layers are clearly important. Radiative dissipation based upon Newton's cooling law is clearly an oversimplification of the problem; nevertheless it allows us to assess the effects of radiative damping on the modal structure. It also enables us to look at the full frequency spectrum and the interaction amongst various modes. Our treatment of the weak field limit has permitted an analysis of the $K-\Omega$ diagram in terms of asymptotic approximations; this has allowed us to understand the nature of the modes in a vertical magnetic field in the presence of radiative exchange. The insight so gained has proved useful in extending the computation to the moderate to strong field case. The transition region lines as observed by cDs on SoHO are capable of diagnosing, Alfv́en, slow and fast magnetoacoustic waves. The Alfv́enic oscillations are essentially velocity oscillations and do not cause any density fluctuations. The compressional modes may however reveal themselves in the form of intensity oscillations through a variation in the emission measure. This fact, together with the oscillations in intensity, allows us to interpret the waves as slow magneto-acoustic in nature. We have computed the frequencies of the modes from the full MAG equation (see Eq. (11)) and found out that for our model atmosphere they correspond to the slow magneto-acoustic modes. The $p_{1}$ and $p_{2}$ mode frequencies fall very well within the observed range (compare Tables 2 and 4). Our observational results very much complement earlier results and provide additional input for the study of the characteristics of the wave modes. Our observations reveal that umbral oscillations are a localized phenomenon, where intensity and velocity both shows a clear peak around $6 \mathrm{mHz}$. In all the wavelet plots, we also notice a smaller peak in the global wavelet spectra and some power in the phase plot around $3 \mathrm{mHz}$, for part of the time sequence, which corresponds to the penumbra. In the theory part of this paper we have shown that the life time of the oscillations are dependent on the relaxation time scale and in some cases these oscillations could be damped within a few oscillations periods as well. Our observations also indicate that the oscillations seems to come in packets with life times of $\sim 10-20 \mathrm{~min}$, which matches fairly well with the damping behavior of our MAG waves. We should also point out that the envelope of these packets do not show exponential decay, as one would expect from the theory, rather the intensity amplitude usually remain sinusoidal. An alternative explanation for the appearance of the packets could be due to the rotation of the sun under the slit than the actual length of the oscillations. We see oscillations for only $20 \mathrm{~min}$ as that may be the time necessary for a source of say, 2 arcsec wide in the 2 arcsec wide slit, to rotate out of the field of view, if the sun is rotating at say, 6 arcsec/hour. In general we find good agreement between the model and observations as far as the duration of oscillation and range of frequency is concerned.

Acknowledgements. DB expresses his gratitude to Profs. S. S. Hasan and Joergen Christensen-Dalsgaard for many valuable discussions which has enabled to develop the theory of the MAG waves. DB wishes to thank the FWO for a fellowship (G.0344.98). EOS is a member of the European PLATON Network. We would like to thank the CDS and EIT teams at Goddard Space Flight Center for their help in obtaining the present data. CDS and EIT are part of SoHO, the Solar and Heliospheric Observatory, which is a mission of international cooperation between ESA and NASA. Research at Armagh Observatory is grant-aided by the N. Ireland Dept. of Culture, Arts and Leisure. This work was supported by PPARC grant PPA/G/S/1999/00055. The original wavelet software was provided by C. Torrence and G. Compo, and is available at URL: http://paos. colorado. edu/research/wavelets/.

\section{References}

Andretta, V., \& Jones, H. P. 1997, ApJ, 489, 375

Babaev, E. S., Dahalilov, N. S., \& Zhugzhda, Yu. D. 1995, Astron. Rep., 39(2), 211

Banerjee, D., Hasan, S. S., \& Christensen-Dalsgaard, J. 1995, ApJ, 451, 825

Banerjee, D., Hasan, S. S., \& Christensen-Dalsgaard, J. 1997, Sol. Phys., 172, 53

Bogdan, T. J., \& Knölker, M. 1989, ApJ, 339, 579

Bogdan, T. J. 2000, Sol. Phys., 192, 373

Bray, R. J., \& Loughhead, R. E. 1974, in The Solar Chromosphere (Chapman \& Hall: London).

Brynildsen, N., Leifsen, T., Kjeldseth-Moe, O., Maltby, P., \& Wilhelm, K. 1999a, ApJ, 511, L121

Brynildsen, Kjeldseth-Moe, O., Maltby, P., \& Wilhelm, K. 1999b, ApJ, 517, L159

Brynildsen, N., Maltby, P., Leifsen, T., Kjeldseth-Moe, O., \& Wilhelm, K. 2000, Sol. Phys., 191, 129

Brynildsen, N., Maltby, P., Fredvik, T., \& Kjeldseth-Moe, O., 2002, Sol. Phys., 207, 259

Bünte, M., \& Bogdan, T. J. 1994, A\&A, 283, 642

Cash, J. R., \& Moore, D. R. 1980, BIT, 20, 44

Doyle, J. G., van den Oord, G. H. J., O'Shea, E., \& Banerjee, D. 1998, Sol. Phys., 181, 51 
Fludra, A. 1999, A\&A, 344, L75

Fludra, A. 2001, A\&A, 368, 639

Harrison, Sawyer, E. C., Carter, M. K., et al. 1995, Sol. Phys., 162, 233

Gurman, J. B., Leibacher, J. W., Shine, R. A., Woodgate, B. E., \& Henze, W. 1982, ApJ, 253, 939

Hasan, S. S., \& Christensen-Dalsgaard, J. 1992, ApJ, 396, 311

Leibacher, J. W., \& Stein, R. F. 1981, in The Sun as a star (NASA), 263

Lites, B. W. 1992, in Sunspots: Theory and observations, ed. J. H. Thomas, \& N. O. Weiss (Dordrecht: Kluwer), 261

Maltby, P., Brynildsen, N., Fredvik, T., Kjeldseth-Moe, O., \& Wilhelm, K. 1999, Sol. Phys., 190, 437

Maltby, P., Brynildsen, N., Kjeldseth-Moe, O., \& Wilhelm, K. 2001, A\&A, 373, L1

Mihalas, D., \& Mihalas, B. W. 1984, in Foundations of Radiation Hydrodynamics (Oxford University Press, New York)
Nemec, A. F., \& Nemec, J. M. 1985, AJ, 90, 2317

O’Shea, E., Banerjee, D., Doyle, J. G., Fleck, B., \& Murtagh, F. 2001, A\&A, 368, 1095

O'Shea, E., Muglach, K., \& Fleck, B. 2002, A\&A, 387, 642

Scheuer, M. D., \& Thomas, J. H. 1981, Sol. Phys., 71, 21

Souffrin, P. 1972, A\&A, 17, 458

Spiegel, E. A. 1957, ApJ, 126, 202

Stix, M. 1970, A\&A, 4, 189

Torrence, C., \& Compo, G. P. 1998, Bull. Amer. Meteor. Soc., 79, 61

Thomas, J. H., Lites, B. W., Gurman, J. B., \& Ladd, E. F. 1987, ApJ, 312,457

Tziotziou, K., Tsiropoula, G., \& Mein, P. 2002, A\&A, 381, 279

Zhugzhda, Yu. D. 1979, Soviet Astron., 23, 42

Zhugzhda, Yu. D., Staude, J., \& Locans, V. 1984, Sol. Phys., 91, 219

Zhukov, V. I. 2002, A\&A, 386, 653 